\title{
On the Land Surface-Atmosphere Coupling and Its Impact in a Single-Column Atmospheric Model
}

\author{
JAN-PETER SCHULZ \\ Danish Meteorological Institute, Copenhagen, Denmark \\ LYDIA DÜMENIL \\ Max-Planck-Institut für Meteorologie, Hamburg, Germany \\ JAN POLCHER \\ Laboratoire de Météorologie Dynamique du CNRS, Paris, France
}

(Manuscript received 3 January 2000, in final form 26 July 2000)

\begin{abstract}
Three versions of the single-column European Centre for Medium-Range Weather Forecasts-Hamburg (ECHAM4) climate model are compared that differ either in the technique of the numerical coupling between land surface and atmosphere or the physical parameterization of the land surface processes. The standard ECHAM4 model utilizes a semi-implicit coupling technique between land surface and atmosphere in a way in which energy at the land surface-atmosphere interface is not conserved. This is a major deficiency. Two new model versions were developed: ECHAM4/IMPL and ECHAM4/SECHIBA. They incorporate an implicit coupling technique that conserves energy. ECHAM4 and ECHAM4/IMPL are identical with respect to all physical parameterizations they apply; the only difference is the coupling. In ECHAM4/SECHIBA, the ECHAM land surface scheme was replaced by SECHIBA (Schématisation des Echanges Hydriques à l'Interface entre la Biosphère et l'Atmosphère). The intercomparison of one-dimensional versions of these three models shows that the energy residual term in ECHAM4 is not negligibly small, but it is rather of the order of the physical fluxes. Biases of more than $1300 \mathrm{~W} \mathrm{~m}^{-2}$ are found due to the coupling technique. These are avoided in ECHAM4/ IMPL, which results in a more pronounced diurnal cycle of surface temperature and generally higher temperature maxima during a warming phase. In an offline intercomparison of the three model versions, using an observational atmospheric forcing dataset, an important impact of the coupling technique on the simulated surface energy cycle is found as well. The turbulent heat fluxes in ECHAM4 tend to be underestimated; their rise in the morning and decrease in the afternoon are delayed. Because of the improved coupling, the turbulent heat fluxes of the implicit models are in better agreement with the observations, especially regarding the phases of their diurnal cycles. Differences between ECHAM4/IMPL and ECHAM4/SECHIBA are mainly found for the simulated surface temperature, which gets closer to the observed radiative temperature for the latter model. Furthermore, the diurnal amplitude of the ground heat flux is increased in ECHAM4/SECHIBA in agreement with the observations.
\end{abstract}

\section{Introduction}

The continental surfaces, including vegetation cover, represent an important component of the earth's climate system. On the one hand, they are the habitat of humanity, which makes it interesting to try to understand the governing processes and living conditions at the land surface and how they may evolve in the future. On the other hand, from the point of view of atmospheric sciences, the land surface and biosphere interact with the

Corresponding author address: Jan-Peter Schulz, Danish Meteorological Institute, Lyngbyvej 100, DK-2100 Copenhagen, Denmark E-mail: jps@dmi.dk lower atmosphere, and they have a significant impact on near-surface meteorological and climatological phenomena.

Atmospheric general circulation models (GCMs) for climate simulations or numerical weather prediction therefore require a realistic description of the land surface processes. The degree of complexity needed for these land surface schemes is not yet completely determined. Numerous numerical experiments have been conducted with GCMs, revealing a sensitivity of the simulated climate to various land surface characteristics. For instance, different components of the global water cycle were found to be sensitive to the available soil moisture or the water-holding capacity of land as well as to land surface albedo or roughness (Shukla and 
Mintz 1982; Milly and Dunne 1994; Charney et al 1977; Sud et al. 1988). Generally, the energy cycle is also affected in these cases, because it is closely coupled to the water cycle.

Apart from these sensitivity experiments, usually including the modification of only one or a few parameter values of the land surface parameterization, some comparison studies have been conducted in which the entire surface scheme was replaced by another one. In most cases one of these models conceptualized the soil hydrological behavior as a simple "bucket" (Manabe 1969). Large differences in the simulated land surface fluxes were found when comparing a bucket and a more complex scheme (e.g., Sato et al. 1989; Stamm et al. 1994). This could be expected because of the shortcomings inherent in the bucket approach such as the neglect of vertical moisture dynamics or a stomatal control of transpiration by vegetation. Mainly because of the latter deficiency, the bucket model commonly tends to compute higher evaporation than the more complex schemes, which contributes to a modified hydrological cycle. There are only very few publications in which two different complex land surface schemes implemented in the same atmospheric GCM are compared, with one of them not just being a simple bucket model. Peylin et al. (1997) compared two simulations with the National Center for Atmospheric Research (NCAR) GCM coupled either to the Biosphere-Atmosphere Transfer Scheme (Dickinson et al. 1986) or the Schématisation des Echanges Hydriques à l'Interface entre la Biosphère et l'Atmosphère (SECHIBA; Ducoudré et al. 1993) land surface model, which were both regarded as complex. SECHIBA is originally the operational land surface scheme of the Laboratoire de Météorologie Dynamique (LMD) GCM. Considerable differences in the simulated climate were found on the regional scale, while on a global scale the effect appeared to be small.

In order to provide a systematic intercomparison of different land surface schemes that are operational in current atmospheric models, the Project for Intercomparison of Land-Surface Parameterization Schemes (PILPS) (Henderson-Sellers et al. 1996) has recently started to implement a number of land surface schemes in the same GCM. Because this is still an ongoing effort, no final results can be reported from that at this time.

Apart from the physical parameterization of the relevant processes, there is another important aspect of the meteorological models, which is the numerical realization of the coupling between land surface and atmosphere and its possible impact on the simulations. Very little attention has been paid to this in the past. Nevertheless, Polcher et al. (1998) stated that "changes to the numerical framework of the land surface scheme may have a strong impact on the behavior of the scheme." Two commonly used (out of four available) coupling techniques are the implicit and the semi-implicit one. The former determines the prognostic variables at the surface and the lowest atmospheric level and the corresponding surface fluxes consistently at the same level in the time-stepping scheme. It is thus energy conserving. The latter uses the atmospheric and surface variables at different subsequent time levels to compute the surface fluxes. This requires a correction of the flux terms after the surface temperature has also been updated. To avoid an energy imbalance, this correction term needs to be taken into account in the calculations at the subsequent time step. Examples for land surface schemes used in GCMs, which are coupled implicitly to their host models, are SECHIBA at LMD or the surface scheme of the U.K. Meteorological Office (Warrilow et al. 1986). On the other hand, the semi-implicit coupling method is used, for instance, in former versions of the European Centre for Medium-Range Weather Forecasts (ECMWF) model (Blondin 1991), or in the ECHAM4 GCM (Roeckner et al. 1996). ECHAM4 is the fourth generation of the ECMWF-Hamburg (ECHAM) GCM, which is a spectral climate model that was developed at the Max-Planck-Institut (MPI) für Meteorologie, Hamburg, Germany. Its land surface scheme will be denoted as ECHAM here. Besides the GCMs, there are also regional climate models (RCMs) incorporating different coupling techniques. Examples for models using the implicit method are the RCMs of the U.K. Meteorological Office (Jones et al. 1995) or Météo-France (Déqué et al. 1998). Semi-implicitly coupled RCMs are, for instance, "HIRHAM" (Christensen et al. 1996) at the Danish Meteorological Institute, or "RACMO" (Christensen and van Meijgaard 1992; van Lipzig et al. 1999) at the Royal Netherlands Meteorological Institute. It would be desirable if differences in the numerical coupling between these GCMs or RCMs, respectively, had only little or no impact on the simulations. However, it will be shown that this is not always the case.

To address this issue, an implicitly coupled version of the ECHAM4 GCM has been developed, here denoted as ECHAM4/IMPL. ECHAM4 and ECHAM4/ IMPL are identical with respect to all physical parameterizations they apply; the only difference is the coupling. This paper compares the one-dimensional versions of these two models that comprise the entire vertical soil-atmosphere column. This allows the study of surface-atmosphere interactions and feedbacks with a focus on the surface exchange processes while avoiding large-scale atmospheric effects such as horizontal advection that would complicate the interpretation of the results. In order to get an estimate of the importance of these numerical aspects their impact is compared with the effect when the ECHAM land surface scheme is replaced by the full SECHIBA. Both schemes are regarded as complex. SECHIBA is implemented in the ECHAM4 GCM using the implicit coupling technique. Therefore, the three GCM versions-ECHAM4, ECHAM4/IMPL, and the so called ECHAM4/SECHI$\mathrm{BA}$ - are a set of models that allows the study of the impact of the numerical coupling in a GCM and the 
comparison with the effects induced by a different land surface representation. These numerical aspects of the land surface coupling have not been analyzed in great detail before. This paper shall provide a first hint that, besides all attempts to improve land surface schemes by better representing and/or including more physical processes, the numerical methods used are of equal importance. For this reason, two different numerical coupling techniques and two different land surface schemes are used here, but this analysis could be extended in future studies by including the other two available coupling methods [explicit and open explicit, cf. Polcher et al. (1998)] and further land surface schemes.

In addition to the intercomparison of their one-dimensional versions, the three models are also tested against observations. For this reason, offline experiments were conducted, using an observational atmospheric forcing dataset. This dataset also includes measurements of the quantities of the surface energy cycle that are utilized for model validation purposes.

This intercomparison study will be extended to the global scale using the full GCMs in a forthcoming paper.

\section{Model descriptions}

The surface energy balance in most meteorological situations is dominated by the total net radiation. Its solar component provides the forcing and the longwave part is usually a sink of energy at the surface. In the absence of water the surface temperature is mainly determined by the balance of these two flux terms. In addition to them, the energy balance includes the turbulent fluxes of sensible and latent heat and the ground heat flux. The energy transfer from the surface to the atmosphere results in a generally heterogeneous temperature distribution, which yields spatial pressure gradients that drive the atmospheric circulation on various scales. Thus, the circulation and climate in general are sensitive to changes in the surface energy fluxes. Because of the close relations of the terms just mentioned, the surface energy balance is considered in GCMs as the link between atmosphere and land surface, with the surface temperature being the key variable.

The meaning of this quantity varies between different land surface schemes. It may be defined as the temperature of the ground, which is either a "layer" of infinitesimal thickness at the surface-atmosphere interface or the upper layer of a soil temperature finite difference scheme. Alternatively, it may describe the temperature of some level within the canopy. This will not be specified further here. The interface between surface and atmosphere may be understood as a "layer" at the surface, which is in contact with the atmosphere. Then the surface energy balance equation is given by

$$
C_{S} \frac{\partial T_{S}}{\partial t}=R_{n}+L E+H+G
$$

where $T_{S}$ is the temperature representing the surface
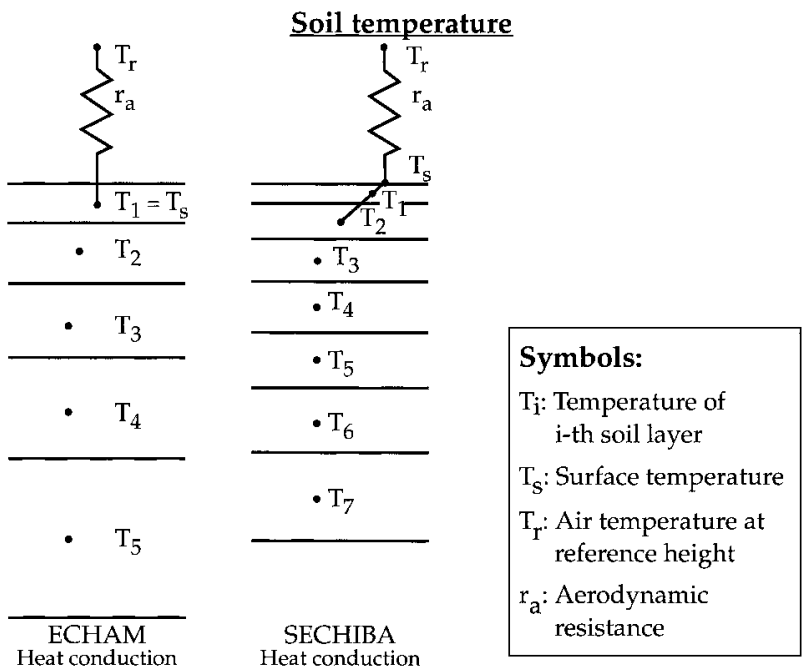

7

layers for soil temperature

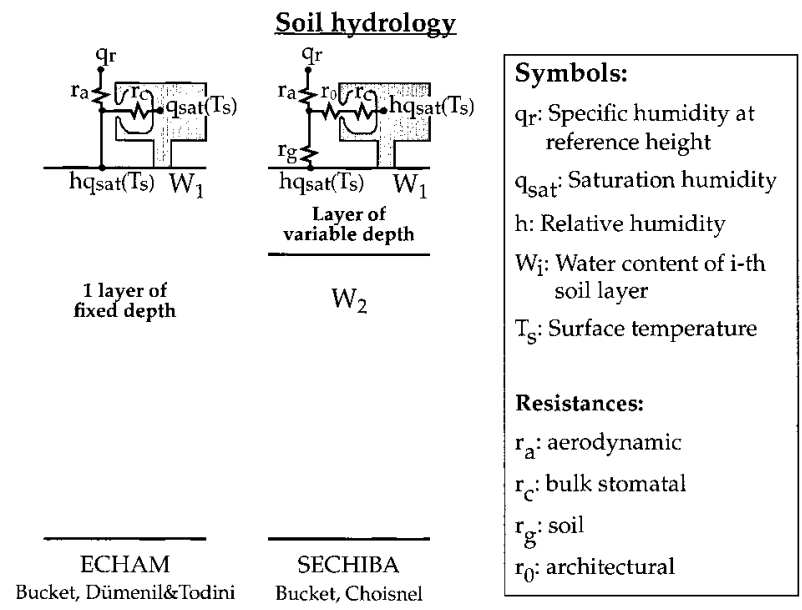

FIG. 1. Schematic representation of the soil temperature layers and the resistances for the calculation of sensible heat fluxes (upper panel) and the soil moisture layers and the resistances for the calculation of latent heat fluxes (lower panel) in ECHAM and SECHIBA.

layer and $C_{S}$ is the heat capacity per unit area. Here, $R_{n}$ is the total surface net radiation, $L E$ the latent heat flux ( $L$ is the latent heat of vaporization or sublimation of water, respectively, and $E$ is the surface moisture flux), $H$ is the sensible heat flux, and $G$ the ground heat flux. The net radiation $R_{n}$ is given by

$$
R_{n}=\left(1-\alpha_{S}\right) R_{\mathrm{sd}}+\epsilon R_{\mathrm{ld}}-\epsilon \sigma T_{S}^{4},
$$

where $R_{\mathrm{sd}}$ and $R_{\mathrm{ld}}$ are the downward shortwave and longwave radiation, respectively. Here, $\alpha_{S}$ is the surface albedo, $\epsilon$ is the thermal emissivity, and $\sigma$ is the StefanBoltzmann constant.

In the following sections the two land surface schemes ECHAM and SECHIBA are described. Figure 1 gives a schematic overview of the schemes. 


\section{a. ЕСHAM}

In the ECHAM model (Roeckner et al. 1992, 1996), the surface fluxes of momentum, heat, and moisture are calculated by means of the classical aerodynamic formulas using the Monin-Obukhov similarity theory (e.g., Stull 1997):

$$
F_{X}=\rho C_{D}|\mathbf{v}|\left(X_{a}-X_{S}\right),
$$

where $\rho$ is the density of air, $C_{D}$ is the drag coefficient for momentum $\left(C_{m}\right)$ or the transfer coefficient for heat and humidity $\left(C_{h}\right)$, and $|\mathbf{v}|$ is the absolute value of the horizontal wind speed. The value of $X_{a}$ may be identified with the atmospheric value of one of the mentioned quantities that are subject to the turbulent exchange, while $X_{S}$ is its surface value. Here, $C_{m}$ and $C_{h}$ are functions of the roughness length, which characterizes the roughness of the surface, and the Richardson number and are calculated by following the scheme of Louis (1979). For evaluating the moisture flux over land, each grid element is divided into four fractions. These fractions are snow cover, water in the interception reservoir, vegetation, and bare soil. This treatment of the vegetation is often referred to as "big leaf" approach, using mean values for parameters like surface resistance, leaf area index or albedo, not taking into account any information on the vegetation distribution within the grid box. The moisture flux $E$ is calculated for each of the four fractions according to the following generic equation:

$$
E=\rho C_{h}|\mathbf{v}| \beta\left[q_{a}-h q_{s}\left(T_{S}, p_{S}\right)\right],
$$

where $q_{s}$ is the saturation-specific humidity at surface temperature $T_{S}$ and pressure $p_{S}$, and $q_{a}$ is the atmospheric specific humidity at a reference level. The values of $\beta$ and $h$ have to be specified for each fraction.

- Snow sublimation and evaporation of water in the interception reservoir are assumed to be at the potential rate, that is, $\beta=h=1$.

- Evaporation from bare soil is limited by the relative humidity $h$ at the surface, which is a function of the relative moisture in the upper soil. The maximum amount of water that is available to bare soil evaporation is the top $10 \mathrm{~cm}$ of the soil water column. For bare soil evaporation, $\beta=1$.

- Based on Sellers et al. (1986), for transpiration, $\beta$ is specified as

$$
\beta=\left[1+\frac{C_{h}|v| r_{c 0}(\mathrm{PAR})}{F\left(W_{S}\right)}\right]^{-1},
$$

including the stomatal resistance $r_{c}\left(\mathrm{PAR}, W_{S}\right)=$ $r_{c 0}(\mathrm{PAR}) / F\left(W_{S}\right)$, which has control over the transpiration from vegetation. Here, $r_{c 0}$ is a function of the photosynthetically active radiation (PAR) and also takes into account the leaf area index (LAI) of the vegetation. The quantity $F\left(W_{S}\right)$ is the water stress factor that depends on the soil water content $W_{S}$ and describes the availability of water in the root zone. It is given by

$$
F\left(W_{S}\right)= \begin{cases}1 & \text { if } W_{\mathrm{cr}} \leq W_{S} \leq W_{S \max } \\ \frac{W_{S}-W_{\mathrm{pwp}}}{W_{\mathrm{cr}}-W_{\mathrm{pwp}}} & \text { if } W_{\mathrm{pwp}}<W_{S}<W_{\mathrm{cr}} \\ 0 & \text { if } 0 \mathrm{~m} \leq W_{S} \leq W_{\mathrm{pwp}} .\end{cases}
$$

The value $W_{S \max }$ is the field capacity, which is defined as the maximum amount of water the soil can hold against gravity over periods of several days. Here $W_{\text {cr }}$ is the critical soil moisture level at which soil moisture stress starts to reduce the transpiration of plants in the model. In the ECHAM4 standard model it is taken as $75 \%$ of $W_{S \max } ; W_{\text {pwp }}$ is the permanent wilting point, taken as $35 \%$ of $W_{S \max }$. It is defined as that soil water level at which the plants are not able anymore to extract further water from the soil. Therefore, when the soil water content is reduced down to $W_{\text {pwp }}$, transpiration stops. For computing the transpiration component, $h$ in (4) is set to 1 .

The total moisture flux is computed as the area weighted average of the four components that are described above.

For the calculation of the soil temperature the concept of heat diffusion is used. The heat conduction equation is solved for five layers over land and land ice, following Warrilow et al. (1986). The temperatures are representative for the middle of each soil layer. The first soil layer in ECHAM is $6.5 \mathrm{~cm}$ deep, the second $25.4 \mathrm{~cm}$, and the thicknesses of the other layers continuously increase with depth. The upper boundary condition is given by the net heat flux (radiative and turbulent) at the surface. At the lower boundary a zero heat flux condition is prescribed to ensure energy conservation in the coupled soil-atmosphere system. In the absence of snow the temperature of the first soil layer $T_{1}$ is regarded as land surface temperature $T_{S}$ (see Fig. 1).

The water budget within the soil is computed for one layer with a prescribed field capacity (bucket model) (Manabe 1969). The governing equation takes precipitation, snowmelt, evapotranspiration, runoff, and drainage into account. Interception of precipitation is treated using one canopy layer. In a simple bucket model surface runoff is produced by overflow when the soil water reservoir is saturated. Here, an advanced approach is chosen that accounts for subgrid scale effects due to the heterogeneity within a grid area (Arno scheme) (Dümenil and Todini 1992). This allows a large fraction of precipitation to infiltrate the soil in the presence of flat terrain, while surface runoff will be more efficient in steep terrain. The drainage rate is taken as a linear function of the soil moisture if it is between 5\% and $90 \%$ of the field capacity. For higher values the drainage rate behaves like a potential function, and for lower values it is equal to zero. Infiltration and drainage do not occur if the soil is frozen. 


\section{b. SECHIBA}

The basic feature of the parameterization of SECHIBA (Ducoudré et al. 1993) is the representation of the transfer of heat and moisture between the atmosphere and the vegetated surface of the earth using a resistance formulation. In analogy to Ohm's law, surface sensible and latent heat fluxes are calculated as follows:

$$
F_{X} \sim \frac{\text { potential difference }}{\text { resistance }}=\frac{\Delta X}{\sum_{i} r_{i}} .
$$

The quantity $X$ may be identified with temperature or specific humidity. The fluxes are driven by $\Delta X$, the gradient of $X$ between surface and a reference level in the atmosphere, and are limited by a sum of resistances $r_{i}$, which may be aerodynamic and/or surface resistances. The formulations of the land surface fluxes as given by (7) and (3) can be converted into each other.

Transpiration and canopy evaporation are computed using up to seven different vegetation types that may be present simultaneously in one grid element (besides bare soil). The moisture flux is calculated separately for each fractional surface cover class, and the total flux of the grid box is determined as an area-weighted average. This technique is called the "mosaic approach" and takes into account the subgrid-scale distribution of the vegetation types.

A difference in SECHIBA to ECHAM is the formulation of the hydrological stress that limits the transpiration. In SECHIBA it is an exponential function of the ratio of the depth of dry soil above the water table and the total depth of the soil moisture layer where the water table is located. The evaporation of foliage water is controlled by the aerodynamic resistance and an architectural resistance, the latter representing the aerodynamic resistance between the leaves and the canopy top (see Fig. 1).

The soil temperature is computed similarly to ECHAM, using the heat conduction equation. Different from ECHAM, SECHIBA utilizes a discretization in seven layers that are not as deep, instead of five. For instance, the upper one is about two-thirds as deep as in ECHAM. A characteristic difference from ECHAM is the method used to determine the surface temperature. In SECHIBA it is calculated using the surface energy balance equation and the soil properties obtained by an extrapolation of heat capacity and ground heat flux toward the surface. This is done by estimating the surface temperature by linear extrapolation of the two uppermost soil layer temperatures toward the surface (cf. Fig. 1). This procedure yields more realistic diurnal variations of the soil temperature profile and ground heat flux close to the surface.

The soil water is stored in two conceptual layers. In contrast to ECHAM, which has a soil moisture reservoir of prescribed fixed depth, in SECHIBA the upper layer has a variable depth (Choisnel scheme) (Choisnel 1977).
If the soil is dry and it starts raining, an upper moisture layer will be created from top to bottom in the soil. When the rain stops, first the soil moisture in the upper layer will be depleted by subsequent evaporation, again from top to bottom until the upper reservoir may disappear. Then evaporation continues from the lower reservoir. This design allows a fast response of bare soil evaporation to precipitation events, as the soil resistance that limits bare soil evaporation directly depends on the depth of dry soil above the water level in the uppermost moisture layer that contains water. Surface runoff only occurs when the soil is saturated; drainage is not computed.

\section{Coupling between land surface and atmosphere}

As noted in section 2, solving the surface energy balance equation [(1)] is the most important task of any land surface scheme, because it closes the energy balance and determines the temperature at the surface. A discretization of (1), using a leapfrog time-stepping scheme, leads to

$$
C_{S} \frac{T_{S}^{t+1}-T_{S}^{t-1}}{2 \Delta t}=R_{n}^{\text {coupl }}+L E^{\text {coupl }}+H^{\text {coupl }}+G^{\text {coupl }},
$$

which gives the evolution of the surface temperature $T_{S}$ over a $2 \Delta t$ time step from time level $t-1$ to $t+1$. The meaning of the other quantities are as in (1). The superscripts of the flux terms on the right-hand side of (8) indicate which numerical coupling technique between land surface and atmosphere is used.

The turbulent fluxes of latent and sensible heat in ECHAM4 are given as follows [notation as in (3) and (4)]:

$$
\begin{aligned}
L E^{\text {coupl }} & =L \rho C_{h}|\mathbf{v}| \beta\left[q_{a}^{j}-h q_{s}\left(T_{S}^{i}\right)\right] \quad \text { and } \\
H^{\text {coupl }} & =\rho C_{h}|\mathbf{v}|\left(s_{a}^{j}-s_{S}^{i}\right) .
\end{aligned}
$$

Here, $s$ is the dry static energy that is defined as

$$
s(z)=C_{p} T(z)+g z,
$$

where $C_{p}$ is the specific heat capacity of air at constant pressure, $T$ is the air temperature at height $z$ above the ground, and $g$ is the acceleration of gravity of the earth. Here, $i$ and $j$ indicate the time levels of the variables. Their choice determines the type of coupling. Two different coupling procedures are discussed here, which are given by the following choices of $i$ and $j$ :

$$
\begin{aligned}
& i=\text { new time step }=t+1, \\
& j=\text { new time step }=t+1: \quad \text { implicit coupling; } \\
& i=\text { previous time step }=t-1, \\
& j=\text { new time step }=t+1: \quad \text { semi-implicit coupling. }
\end{aligned}
$$

In the next two sections it is described how the two different coupling techniques are applied to the surface energy balance equation. Because the focus is on the coupling between land surface and atmosphere, and in 
order to simplify the equations, the expression for the ground heat flux will not be given. It is calculated from a heat diffusion equation in the soil, which is of the same type as the one used for the vertical diffusion in the lower atmosphere (see the appendix). Two other methods are also available but they are not used here because the first one (explicit) is computationally too expensive and does not guarantee a unique solution and the other one (open explicit) has numerical stability problems in GCMs. For further information the reader is referred to Polcher et al. (1998).

\section{a. Implicit coupling}

The objective of this method is to provide numerical stability of the solution of the equation and to calculate the prognostic variables and fluxes synchronously at the same level of time. This leads to a consistent solution for the atmospheric profiles of temperature and humidity, including the surface conditions and the vertical heat fluxes.

In order to apply this method to the surface energy balance equation, the atmospheric values of temperature and humidity, used in the formulation of the surface heat fluxes, need to be known. As shown in the appendix, these quantities are related to the surface conditions by (A7) (see also Richtmyer and Morton 1967). In this equation the prognostic variable for level $k$ is $X_{k}^{*} / \alpha$. For the clarity of the implicit treatment of the surface energy balance equation, here we prefer to use the relations

$$
\begin{aligned}
& q_{a}^{t+1}=E_{q, N+1 / 2}^{t-1} q_{s}\left(T_{S}^{t+1}\right)+F_{q, N+1 / 2}^{t-1} \text { and } \\
& s_{a}^{t+1}=E_{s, N+1 / 2}^{t-1} s_{S}^{t+1}+F_{s, N+1 / 2}^{t-1},
\end{aligned}
$$

which give, similar to (A7), the variables at the lowest atmospheric level as functions of the surface conditions, but here both at time step $t+1$. The surface energy balance equation can also easily be formulated using the $X_{k}^{*} / \alpha$ variables, but here the description of the numerical scheme shall be kept as simple as possible. Thus, the expressions for the total net radiation [cf. (2)] and the latent and sensible heat fluxes [cf. (9), (10), (12), and (13)] become

$$
\begin{gathered}
R_{n}^{\mathrm{impl}=}\left(1-\alpha_{S}\right) R_{\mathrm{sd}}+\epsilon R_{\mathrm{ld}}-\epsilon \sigma\left(T_{S}^{t+1}\right)^{4}, \\
L E^{\mathrm{impl}=} L \rho C_{h}|\mathbf{v}| \beta\left\{\left[E_{q, N+1 / 2}^{t-1} q_{S}\left(T_{S}^{t+1}\right)+F_{q, N+1 / 2}^{t-1}\right]\right. \\
\left.\quad-h q_{s}\left(T_{S}^{t+1}\right)\right\}, \quad \text { and } \\
H^{\mathrm{impl}=} \rho C_{h}|\mathbf{v}|\left[\left(E_{s, N+1 / 2}^{t-1} s_{S}^{t+1}+F_{s, N+1 / 2}^{t-1}\right)-s_{S}^{t+1}\right] \\
=\rho C_{h}|\mathbf{v}|\left[\left(E_{s, N+1 / 2}^{t-1} C_{p} T_{S}^{t+1}+F_{s, N+1 / 2}^{t-1}\right)\right. \\
\left.-C_{p} T_{S}^{t+1}\right] .
\end{gathered}
$$

Inserting these expressions in (8) gives a fully implicit equation for the surface energy balance

$$
\begin{aligned}
C_{S} & \frac{T_{S}^{t+1}-T_{S}^{t-1}}{2 \Delta t} \\
= & \left(1-\alpha_{S}\right) R_{\mathrm{sd}}+\epsilon R_{\mathrm{ld}}-\epsilon \sigma\left(T_{S}^{t+1}\right)^{4} \\
& +L \rho C_{h}|\mathbf{v}| \beta\left\{\left[E_{q, N+1 / 2}^{t-1} q_{s}\left(T_{S}^{t+1}\right)+F_{q, N+1 / 2}^{t-1}\right]-h q_{s}\left(T_{S}^{t+1}\right)\right\} \\
& +\rho C_{h}|\mathbf{v}|\left[\left(E_{s, N+1 / 2}^{t-1} C_{p} T_{S}^{t+1}+F_{s, N+1 / 2}^{t-1}\right)-C_{p} T_{S}^{t+1}\right] \\
& +G^{\mathrm{impl}} .
\end{aligned}
$$

Because an equation is desired that is linear in $T_{S}$, the nonlinear terms are replaced by their truncated Taylor expansions. These terms are the upward longwave radiation and the saturated specific humidity:

$$
\begin{aligned}
\epsilon \sigma\left(T_{S}^{t+1}\right)^{4} & \approx \epsilon \sigma\left(T_{S}^{t-1}\right)^{4}+4 \epsilon \sigma\left(T_{S}^{t-1}\right)^{3}\left(T_{S}^{t+1}-T_{S}^{t-1}\right) \\
& =: R_{\mathrm{lu}}^{t+1} \text { and } \\
q_{S}\left(T_{S}^{t+1}\right) & \approx q_{S}\left(T_{S}^{t-1}\right)+\left.\frac{\partial q_{S}}{\partial T_{S}}\right|_{T_{S}^{t-1}}\left(T_{S}^{t+1}-T_{S}^{t-1}\right) .
\end{aligned}
$$

With this linearization, (17) can be resolved for $T_{S}^{t+1}$. Subsequently the back substitution can be performed, which yields the atmospheric profiles of temperature and humidity (see the appendix). The latent and sensible heat flux are calculated from (15) and (16). These expressions are incorporated in the surface energy balance equation (17), which ensures energy conservation in the coupled system, since the GCM consistently receives the same energy fluxes as used in the land surface scheme.

Because the radiation module in ECHAM4 has already completed its calculations before the vertical diffusion component starts operation, care has to be taken of the upward longwave radiation concerning the energy conservation. Once the surface energy balance (17) is solved, the so-called radiative temperature $T_{\text {rad }}$ will be computed, which is related to $R_{\mathrm{lu}}^{t+1}$ [cf. (18)] by

$$
R_{\mathrm{lu}}^{t+1}=\epsilon \sigma\left(T_{\mathrm{rad}}^{t+1}\right)^{4} .
$$

Passing the radiative temperature to the radiation scheme will ensure that the time-averaged balance of the longwave radiative fluxes is closed.

In the model versions ECHAM4/IMPL and ECHAM4/SECHIBA the soil temperature schemes are also coupled implicitly to the surface energy balance. This leads to a closed set of equations that describes the vertical heat exchange in the entire column from the top of the planetary boundary layer to the bottom of the soil.

The implicit method as used here is numerically stable and it conserves energy, because the surface fluxes are consistent with the atmospheric profiles of temperature and humidity. Problems may arise, for instance, if the water supply is not sufficient for the calculated evaporation. This may happen because the fluxes are computed implicitly in (17), but with explicit coefficients. Therefore, a limiting condition like a soil moisture stress 
formulation may fail in effectively reducing excessive evaporation. This problem is avoided by a predictorcorrector method for the coefficients, as implemented in SECHIBA. In general, an explicit calculation of the coefficients may become problematic if their value varies rapidly in time. This may be the case for the transfer coefficient $C_{h}$ when the stability regime changes. But, for typical GCM applications using time steps of 30 min or less, an explicitly computed $C_{h}$ can generally be safely applied and will lead to reasonably accurate solutions of the turbulent heat fluxes.

\section{b. Semi-implicit coupling}

In contrast to the implicit coupling, in this method the surface energy fluxes are calculated using the surface temperature value at the previous time step [cf. (9) and (10)]. Thus, the following equations are obtained for the fluxes [see (14), (15), and (16)]:

$$
\begin{gathered}
R_{n}^{\mathrm{semi}}=\left(1-\alpha_{S}\right) R_{\mathrm{sd}}+\epsilon R_{\mathrm{ld}}-\epsilon \sigma\left(T_{S}^{t-1}\right)^{4}, \\
L E^{\mathrm{semi}}=L \rho C_{h}|\mathbf{v}| \beta\left\{\left[E_{q, N+1 / 2}^{t-1} q_{s}\left(T_{S}^{t-1}\right)+F_{q, N+1 / 2}^{t-1}\right]\right. \\
\left.-h q_{s}\left(T_{S}^{t-1}\right)\right\}, \quad \text { and } \\
H^{\mathrm{semi}}=\rho C_{h}|\mathbf{v}|\left[\left(E_{s, N+1 / 2}^{t-1} C_{p} T_{S}^{t-1}+F_{s, N+1 / 2}^{t-1}\right)\right. \\
\left.-C_{p} T_{S}^{t-1}\right] .
\end{gathered}
$$

These formulations for the latent and sensible heat flux [(22) and (23)] are used to close the vertical diffusion scheme that computes the new atmospheric temperature and humidity profiles. In order to provide numerical stability when solving the surface energy balance equation [(8)], truncated Taylor expansions of the flux terms with respect to the (fast varying) surface temperature are used:

$$
\begin{aligned}
C_{S} \frac{T_{S}^{t+1}-T_{S}^{t-1}}{2 \Delta t}= & R_{n}^{\mathrm{semi}}+\left.\frac{\partial R_{n}}{\partial T_{S}}\right|_{T_{S}^{t-1}}\left(T_{S}^{t+1}-T_{S}^{t-1}\right) \\
& +L E^{\mathrm{semi}}+\left.\frac{\partial L E}{\partial T_{S}}\right|_{T_{S}^{t-1}}\left(T_{S}^{t+1}-T_{S}^{t-1}\right) \\
& +H^{\mathrm{semi}}+\left.\frac{\partial H}{\partial T_{S}}\right|_{T_{S}^{t-1}}\left(T_{S}^{t+1}-T_{S}^{t-1}\right) \\
& +G^{\mathrm{semi}} .
\end{aligned}
$$

In ECHAM4 this equation is solved after the radiation and the vertical diffusion schemes have calculated the surface energy fluxes using (21), (22), and (23). Therefore, the fluxes that are balanced by the surface energy equation [(24)], and which correspond to the new value of the surface temperature, are different from those received by the atmosphere. This means that the energy balance at the surface is not closed in the model. It is not implied here that this is a principal deficiency of the semi-implicit method, but the imbalance has to be taken into account in the temperature calculation at the subsequent time step. This is not done in the standard ECHAM4 GCM. The surface residual energy, given by the sum of the three Taylor expansion terms in (24), is assumed to be small, but, as shown later, this may not necessarily be the case in all situations. By design, the semi-implicit coupling only allows the surface temperature to feed back on the atmosphere at the subsequent time step, rather than simultaneously. More information on the coupling techniques can be found in Polcher et al. (1998).

\section{Single-column model studies}

Global-scale GCMs are generally very complex and incorporate many degrees of freedom. Therefore, the results of GCM sensitivity experiments are often difficult to interpret; thus they remain very general. In contrast to this, when forcing the land surface scheme with an atmospheric dataset in offline simulations, feedback effects between surface and atmosphere are totally excluded. This limits the transferability of the findings to the coupled land-atmosphere system. A compromise between these two cases is to use a coupled one-dimensional (1D) model. Here, the entire vertical column (of a GCM) is simulated, including the atmosphere and the soil, at one point, considering all physical processes included in the global model, for example, radiation, vertical diffusion, formation of stratiform and convective precipitation, and land surface processes. This allows the study of surface-atmosphere exchange processes, including feedbacks, while no large-scale atmospheric effects such as horizontal advection complicate the interpretation of the results. In this section, results from the one-dimensional versions of the ECHAM4, ECHAM4/IMPL, and ECHAM4/SECHIBA GCMs are presented.

A 1D simulation for July at a site in central Europe $\left(54^{\circ} \mathrm{N}, 11^{\circ} \mathrm{E}\right)$ was performed with each of the three schemes, using the same initialization, taken from a previous ECHAM4 GCM simulation, and equivalent land surface characteristics. An advective forcing was not applied. The vegetation is grass with a coverage of $80 \%$. For the field capacity in ECHAM4 the typical value $0.23 \mathrm{~m}$ is chosen, which is associated with a wilting point [i.e., $35 \%$ of the field capacity in the model, cf. (6)] of $0.08 \mathrm{~m}$. Therefore, the maximum plant-available soil moisture, that is, the difference between field capacity and wilting level, amounts to $0.15 \mathrm{~m}$. In the model structure of SECHIBA the wilting level is at zero by definition. Thus, the active soil moisture volume is equal to the maximum plant-available soil moisture, which is also $0.15 \mathrm{~m}$ for grass. The three models were initialized at $7.7 \%$ of the plant-available soil moisture, which represents very dry conditions. Here, some important characteristic aspects of the numerical and physical differences between the three models can be demonstrated.

Figure 2 shows the diurnal variations of the surface energy fluxes of ECHAM4 for the first three days of 


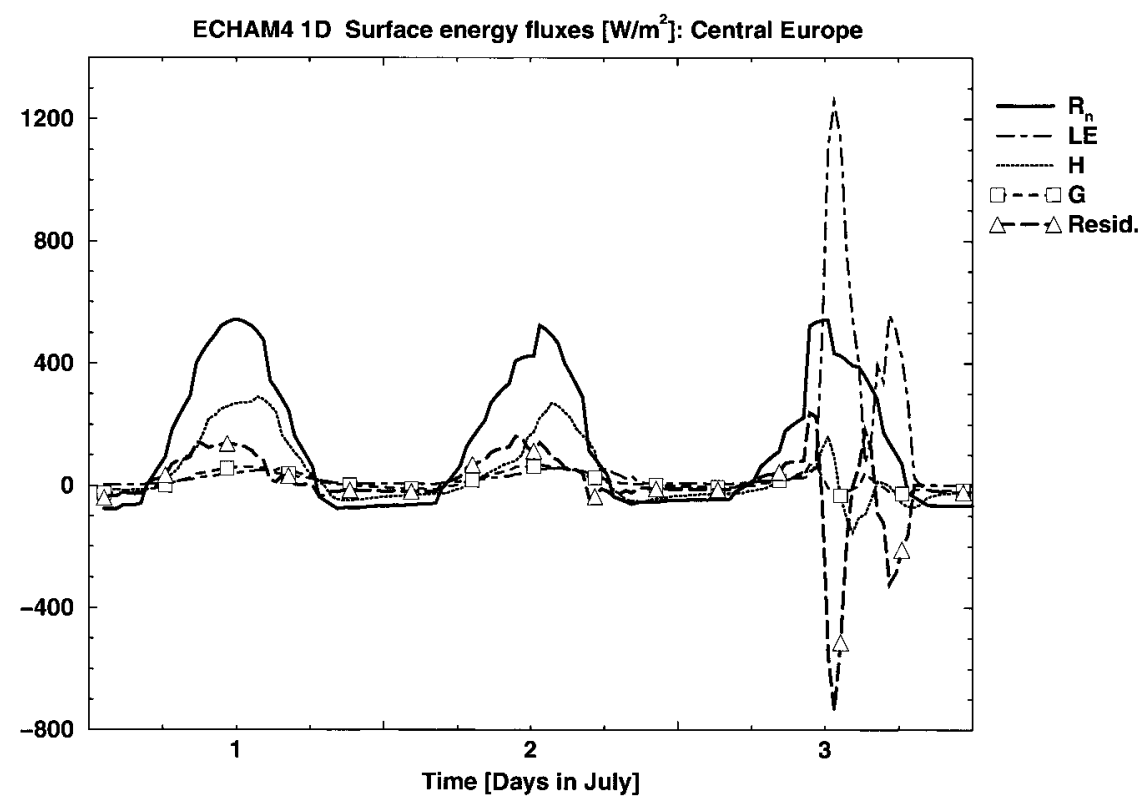

FIG. 2. Diurnal cycles of total net radiation $\left(R_{n}\right)$, latent heat flux $(L E)$, sensible heat flux $(H)$, ground heat flux $(G)$, and the surface energy residual term as simulated by ECHAM4 1D from 1 to $3 \mathrm{Jul}$ at a site in central Europe. Note that the residual is not small, which was assumed, but is of the order of the physical fluxes or even much higher, leading to significantly erroneous flux simulations (e.g., on $3 \mathrm{Jul}$ ).

the $1 \mathrm{D}$ simulation. The total net radiation is characterized by periodically similar diurnal cycles with maxima of about $550 \mathrm{~W} \mathrm{~m}^{-2}$ at noon and minima of about -80 $\mathrm{W} \mathrm{m}^{-2}$ at the beginning of the nights. This energy should be balanced by the other flux terms of the surface energy equation and a change in surface temperature [cf. (1)]. Because the soil is very dry and there is almost no water in the interception reservoir, the evapotranspiration and thus latent heat flux is small on days 1 and 2 . Therefore, the sensible heat flux dominates the flux terms that balance the net radiation. The plotted surface energy residual is the sum of the three Taylor expansion terms with respect to surface temperature in (24) (cf. section $3 b)$. When formulating the equations for the semi-implicit coupling, it was assumed that this residual term, which represents an error in the surface-atmosphere energy balance, would be small in comparison with the physical fluxes. However, in periods of large variations of the surface temperature it can become very large [by definition, cf. (24)]. This can be seen in Fig. 2, where on days 1 and 2 the residual is actually of a magnitude similar to the fluxes. The fact that it is positive reflects an energy loss at the surface due to the numerics. On day 3 the residual term shows a large negative peak at noon, while a very large positive peak appears in the latent heat flux, lasting for several hours and reaching a maximum value of about $1250 \mathrm{~W} \mathrm{~m}^{-2}$, a completely unrealistic value. The energy flux is more than twice as high as that of the total net radiation. The ground heat and the sensible heat flux are inverted during this period, but the major part of the energy is provided by the residual term, which means it has no physical source but is artificially created by the numerics! Later in the afternoon of the same day there is another similar but smaller peak in the latent heat flux.

In order to get a better understanding of this behavior, the moisture source of this enormous evapotranspiration shall be analyzed. Figure 3 shows the different components that are aggregated to the total latent heat flux. During the first two days transpiration dominates, showing diurnal cycles of a realistic order of magnitude. Bare soil evaporation is zero during the entire period of simulation because of drought stress. The third day also starts with transpiration, which is rapidly superposed by two peaks of canopy evaporation at noon and in the second half of the day. Why is the canopy evaporation so heavily overestimated? Figure 4 compares the total precipitation and evapotranspiration simulated by ECHAM4 on these three days. Although there is not a large amount of rain on the first two days, two convective precipitation events take place on the third day coinciding with the canopy evaporation peaks, the higher one reaching a maximum value of about $42 \mathrm{~mm}$ day $^{-1}$. This implies, once the convective rain starts, the interception reservoir is moistened, allowing for canopy evaporation, which, in turn, triggers convective precipitation. The result is a positive feedback, which may be realistic and an important effect at certain locations, for example, in the tropical rainforest. However, in the example presented here, the latent heat flux is highly overestimated because of the erroneous residual energy term, which is part of the semi-implicit coupling scheme im- 


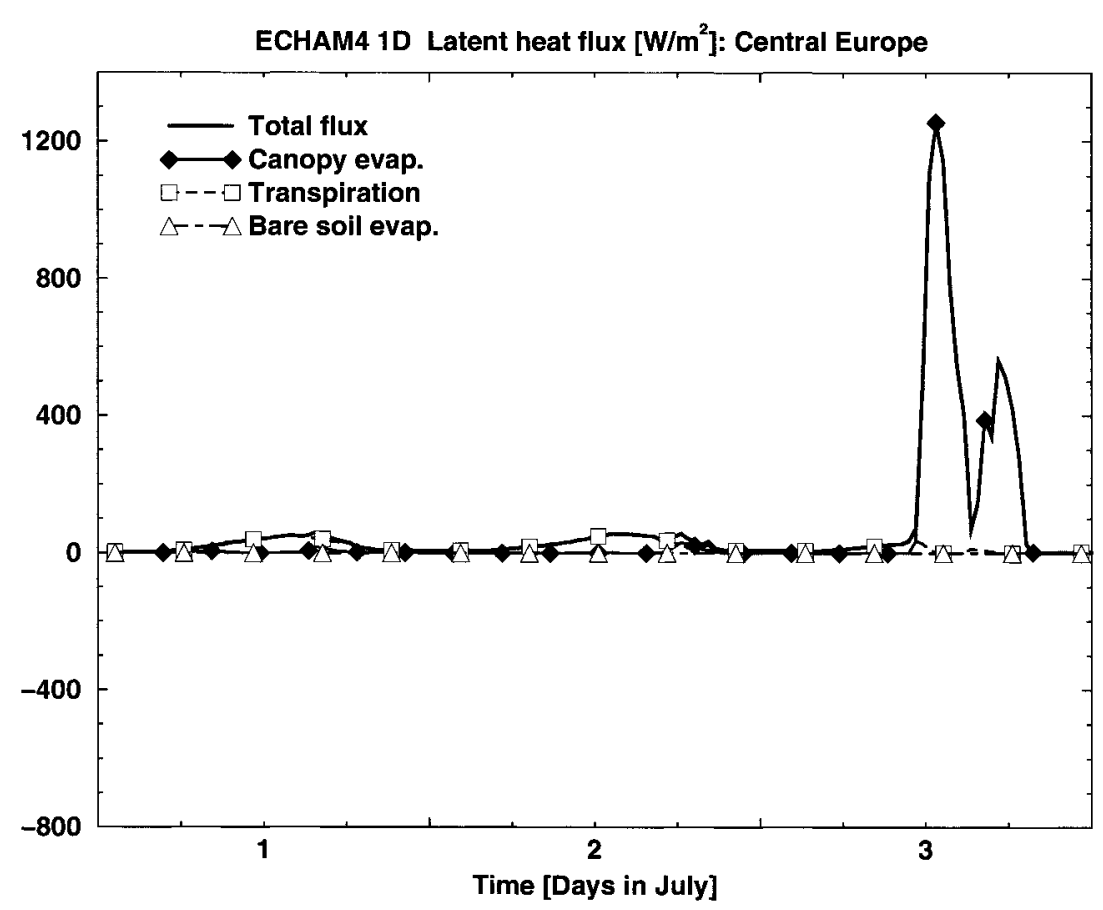

FIG. 3. Contributions of canopy evaporation, transpiration, and bare soil evaporation to the total latent heat flux as simulated by ECHAM4 1D from 1 to 3 Jul at a site in central Europe.

plemented in the standard ECHAM4 GCM. This is a clear model deficiency and shows that great care needs to be taken in the choice of numerical methods applied.

These results from the standard ECHAM4 presented above shall be compared with the case when an implicit coupling scheme is used, that is, with ECHAM4/IMPL.
Figure 5 shows the diurnal variations of the surface energy fluxes of ECHAM4/IMPL 1D from 1 to 3 July. The total net radiation evolves similarly to ECHAM4 with diurnal cycles similar in shape and amplitude. The obvious difference to the semi-implicit model is the absence of a residual energy term. A comparison of Figs.

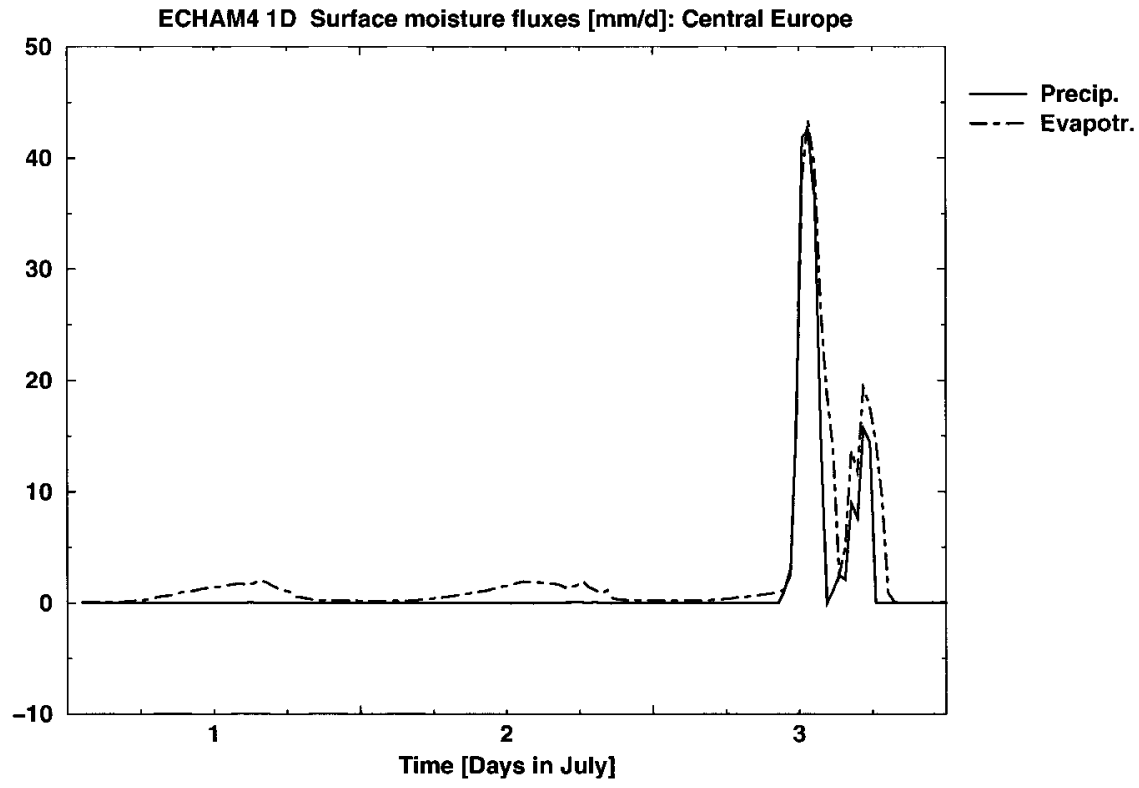

FIG. 4. Diurnal cycles of precipitation and evapotranspiration as simulated by ECHAM4 1D from 1 to $3 \mathrm{Jul}$ at a site in central Europe. 


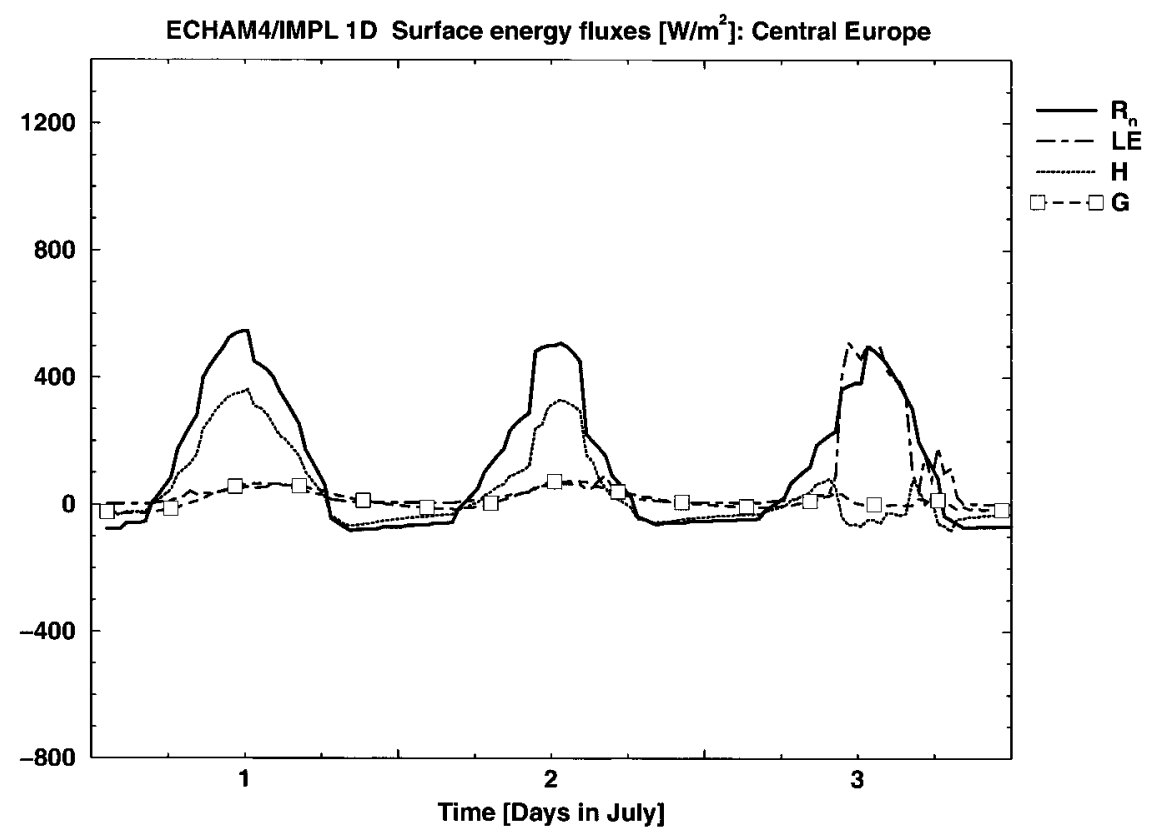

FIG. 5. Diurnal cycles of total net radiation $\left(R_{n}\right)$, latent heat flux $(L E)$, sensible heat flux $(H)$, and ground heat flux $(G)$ as simulated by ECHAM4/IMPL 1D from 1 to $3 \mathrm{Jul}$ at a site in central Europe.

2 and 5 shows that this energy that is not lost to the residual term anymore would appear to contribute mainly to the sensible heat flux on days 1 and 2 in the implicit model. This appears to be reasonable for the prevailing dry conditions. On day 3 the convective precipitation event appears again (see Fig. 6), but in comparison with that in ECHAM4 it is much smaller. As in ECHAM4, a positive feedback between convective precipitation and canopy evaporation occurs, but here the energy available for the latent heat flux is much better controlled. It is provided or compensated by the other surface energy fluxes or a change of surface temperature. As seen in Fig. 5, the total net radiative flux provides an upper limit for the latent heat flux on day 3 , and when this limit is exceeded it is compensated by the sensible heat flux, which becomes negative at noon on

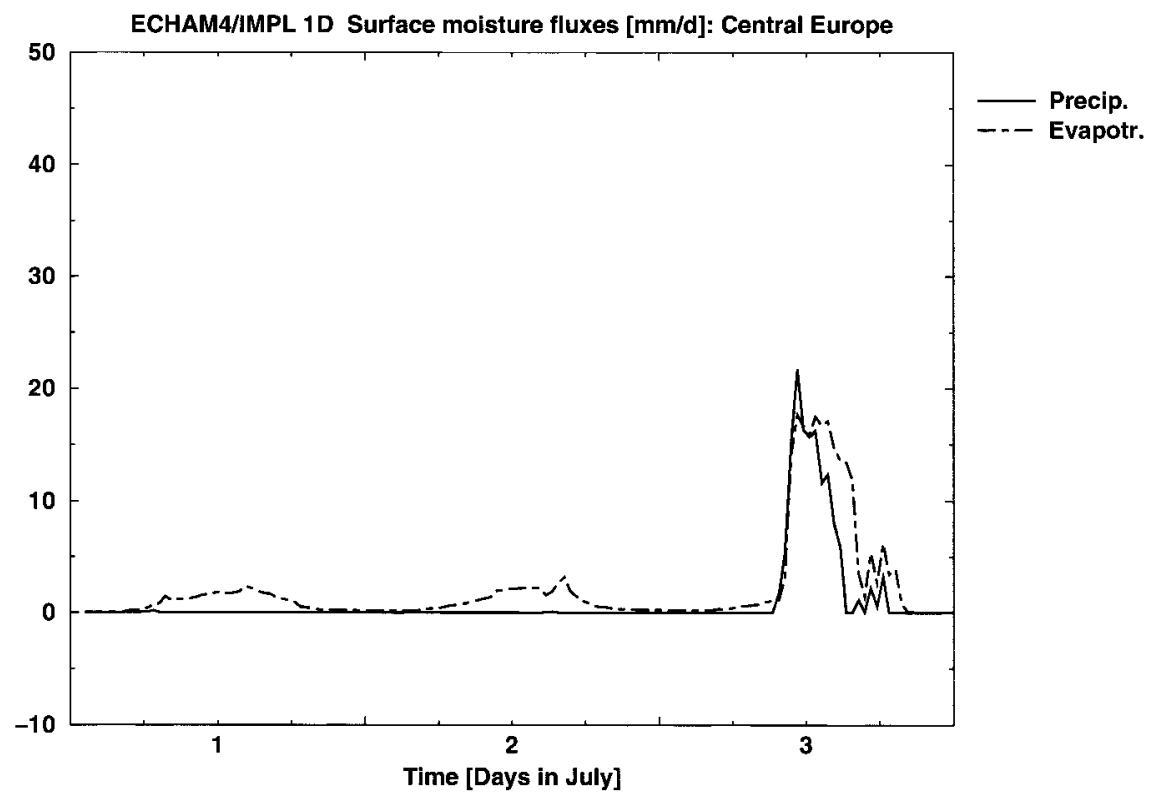

FIG. 6. Diurnal cycles of precipitation and evapotranspiration as simulated by ECHAM4/IMPL 1D from 1 to 3 Jul at a site in central Europe. 


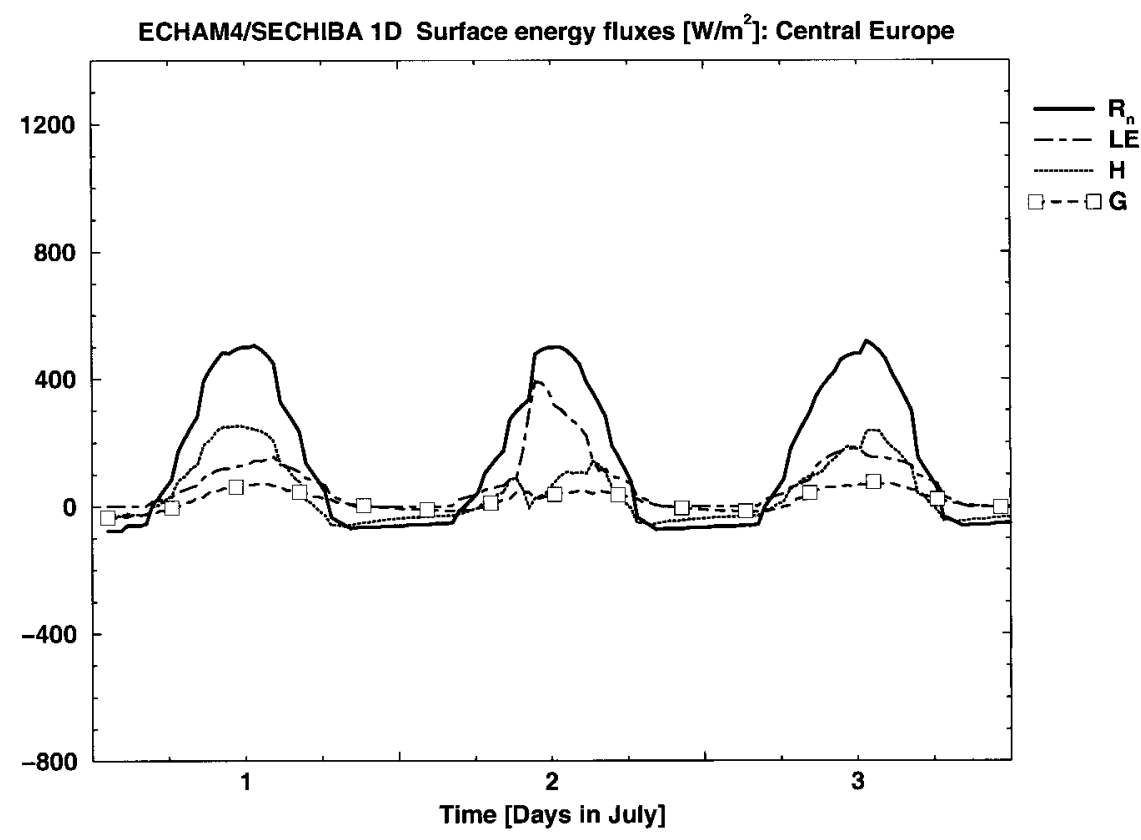

FIG. 7. Diurnal cycles of total net radiation $\left(R_{n}\right)$, latent heat flux $(L E)$, sensible heat flux $(H)$, and ground heat flux $(G)$ as simulated by ECHAM4/SECHIBA 1D from 1 to $3 \mathrm{Jul}$ at a site in central Europe.

day 3 , and the ground heat flux. In addition, the latent heat flux causes a distinct cooling of the surface, as shown later. Thus, the relations between the different quantities of the energy and moisture cycles just presented are physically correct. Because of the evaporation-precipitation feedback this also has implications for the precipitation. As shown, the semi-implicitly coupled ECHAM4 simulates an unrealistic high evaporation peak on day 3 , which is associated with a maximum precipitation of about $42 \mathrm{~mm}$ day $^{-1}$. In ECHAM4/IMPL the corresponding precipitation maximum value is at about $21 \mathrm{~mm} \mathrm{day}^{-1}$, a much more realistic value under the conditions at this location.

The simulated energy fluxes in ECHAM4/SECHIBA, shown in Fig. 7, look essentially similar to those in ECHAM4/IMPL with respect to their orders of magnitude and the energy partitioning between the different fluxes. This is expected because the numerical coupling scheme is the same and the thermodynamic parts are very similar in both models. Furthermore, one of the main features of SECHIBA that makes it different to ECHAM, the mosaic approach for the treatment of the vegetation within a grid cell, does not apply here because only one biome type is used in these experiments. Therefore, the main differences between these schemes in this case are the soil hydrologic scheme together with the soil moisture stress formulation. On the first day the evapotranspiration and thus the associated latent heat flux are higher in ECHAM4/SECHIBA in comparison with ECHAM4/IMPL; as a consequence, the sensible heat flux is lower. This is an artifact of the soil moisture initialization in combination with the different soil moisture stress term. Because of the enhanced transpiration, convective precipitation is triggered on the second day already (cf. Fig. 8), that is, one day earlier than in ECHAM4/IMPL. As in the two other experiments, this is accompanied by a peak in canopy evaporation. The maximum precipitation value is similar to that in ECHAM4/IMPL.

As expected, the higher latent heat flux in ECHAM4/ SECHIBA on the first two days causes a lower surface temperature, best visible on day 2 (Fig. 9). On the third day a rapid cooling is seen in ECHAM4 as result of the peak in latent heat flux around noon (cf. Fig. 2). ECHAM4/IMPL shows a cooling on the third day as well, but not so pronounced. On days 1 and 2, when the energy partitioning between the different surface fluxes in ECHAM4 and ECHAM4/IMPL is similar (apart from the residual term in ECHAM4), the surface temperature of the two models shows a similar evolution as well. However, there is a tendency for the temperature maxima to be slightly higher in ECHAM4/IMPL. This is due to the fact that in ECHAM4 some energy is lost to the energy residual. In ECHAM4/IMPL, this energy is mainly used, in this example, to increase maximum surface temperature and sensible heat flux, as discussed earlier.

Another feature of the ECHAM4 parameterization shall be mentioned here, concerning the soil moisture budget. Figure 10 shows the evolution of the relative plant-available soil moisture of the three models during the entire 30-day period of simulation. They are initialized at $7.7 \%$. Because on average, evapotranspiration exceeds precipitation in all three models, the soil water 


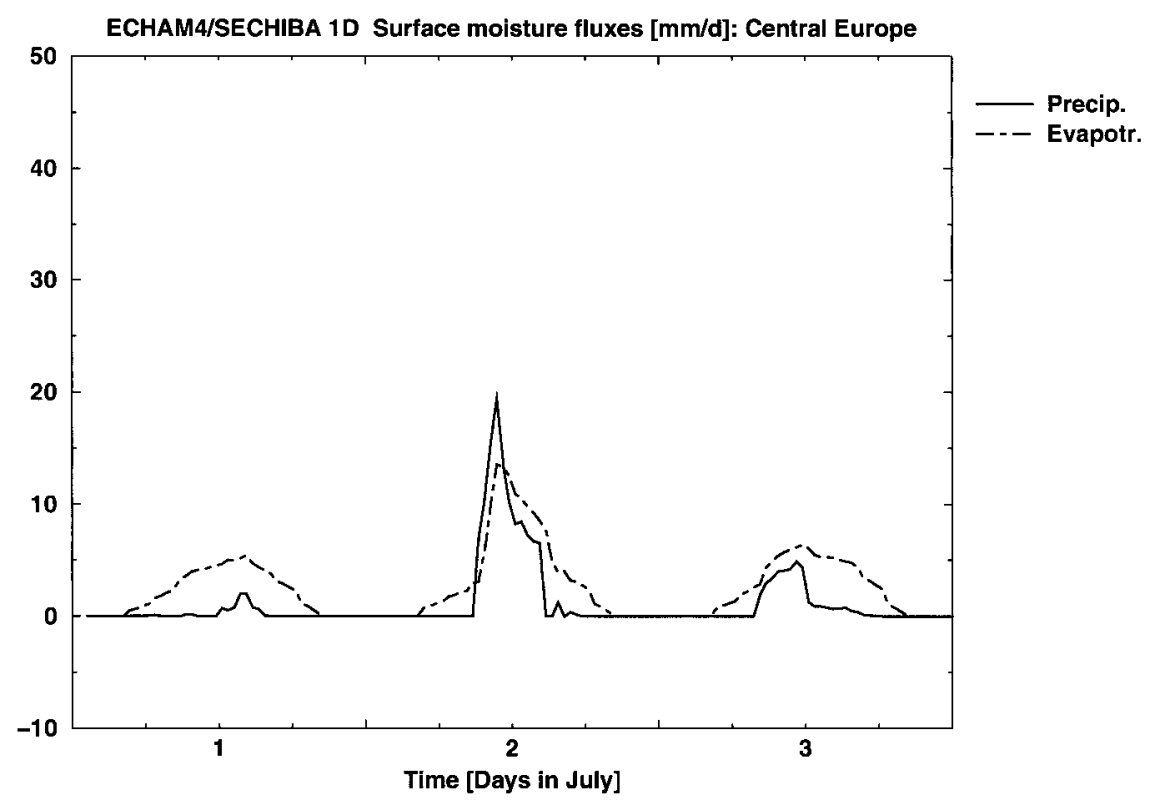

FIG. 8. Diurnal cycles of precipitation and evapotranspiration as simulated by ECHAM4/ SECHIBA 1D from 1 to $3 \mathrm{Jul}$ at a site in central Europe.

content decreases gradually in a similar way until the zero level is reached, which represents the wilting point. This is defined as that soil water level below which transpiration is zero. At this point SECHIBA remains at a zero level while the water content of the other models further decreases! Bare soil evaporation is zero in ECHAM during this simulation because of drought stress. Therefore, only transpiration would be able to reduce the soil moisture in ECHAM4 and ECHAM4/ IMPL according to the equations. However, transpiration stops at the wilting point because of the water stress factor [see Eq. (6)]. The reason for the further-decreasing soil water content is a conceptual inconsistency in ECHAM that allows the removal of additional water

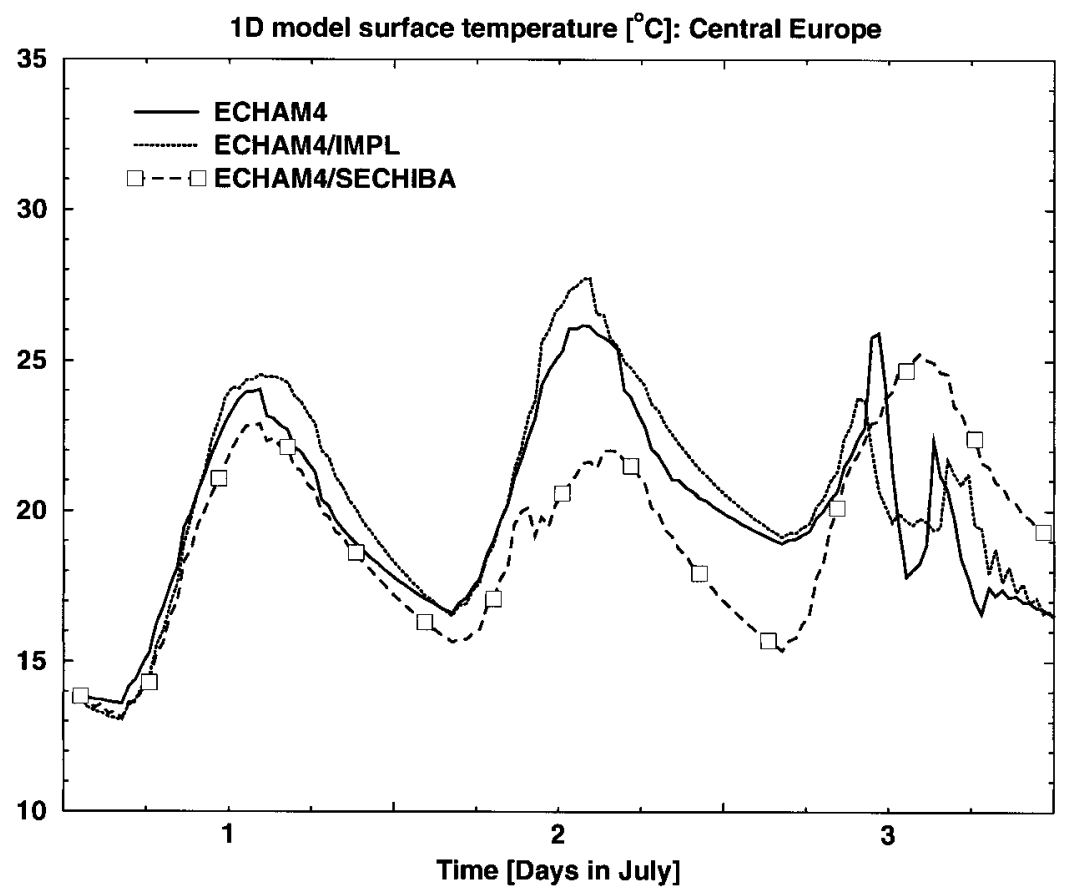

FIG. 9. Diurnal cycles of surface temperature as simulated by ECHAM4, ECHAM4/ IMPL, and ECHAM4/SECHIBA 1D from 1 to $3 \mathrm{Jul}$ at a site in central Europe. 


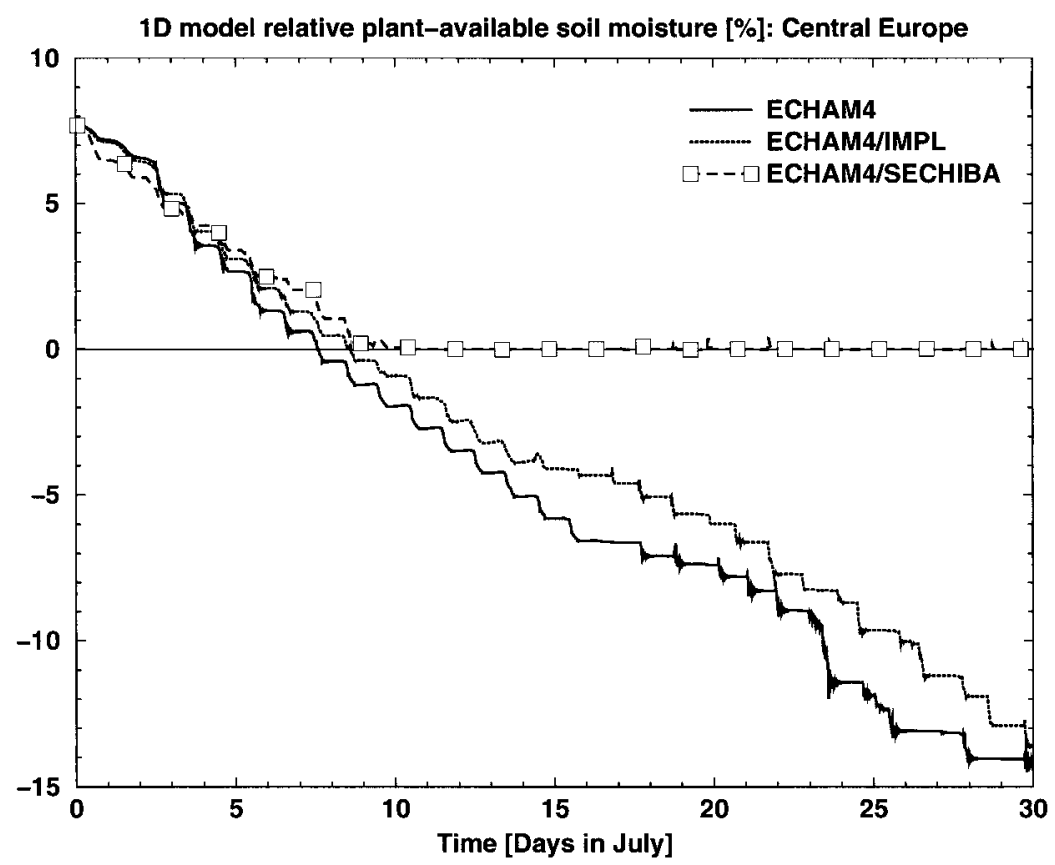

FIG. 10. Evolution of relative plant-available soil moisture as simulated by ECHAM4, ECHAM4/IMPL, and ECHAM4/SECHIBA 1D from 1 to $30 \mathrm{Jul}$ at a site in central Europe. The 0\%-level indicates the wilting point. The ECHAM4 and ECHAM4/IMPL values below it are physically unrealistic, and they occur because of a conceptual inconsistency in the parameterization (cf. text).

from the soil when required, which occurs whenever the calculated canopy evaporation exceeds the available water in the interception reservoir. This was not modified in ECHAM4/IMPL because the idea is to study the impact of the different numerical coupling in ECHAM4/ IMPL while the physical equations remain completely unchanged.

This phenomenon points to principal difficulties that arise because explicit coefficients are used for computing the surface fluxes. In ECHAM this can lead to moisture fluxes that are higher than the actual content of the respective reservoir (i.e., snowpack, water in the interception reservoir, or soil water). In SECHIBA such an effect is avoided by a predictor-corrector method that reduces the canopy evaporation according to the available water on the canopy.

Apart from that, because of a different formulation of the soil moisture stress SECHIBA additionally allows some bare soil evaporation in this situation. This can happen after heavy rain showers when the upper soil moisture layer is filled. This is the case on day 2 , for instance (cf. Fig. 8). Furthermore, in the model structure of SECHIBA the wilting level is at zero by definition. This appears to be a reasonable approach, since below it transpiration is zero and bare soil evaporation is very low or also zero as in ECHAM in this example. An exception is when there is water in the upper soil while the total soil column is very dry on average, but this case is well-treated by the SECHIBA hydrologic scheme.

In order to get an impression of the evolution of the surface temperature in the three models during the simulation, Fig. 11 shows the period from 16 to 19 July. On day 16 the temperatures in ECHAM4 and ECHAM4/ IMPL increase similarly in the morning, but at noon ECHAM4's temperature suddenly decreases while ECHAM4/IMPL's further rises. The reason is a peak in latent heat flux, enlarged by an energy supply from the residual (see Fig. 12). This effect, and the energy that is lost through the residual every day, tend to diminish the temperature increase in ECHAM4 during a warming phase, especially under dry conditions. Therefore, ECHAM4/IMPL shows a more pronounced diurnal temperature amplitude and generally higher temperature maxima during a warming phase. The surface temperature in ECHAM4/SECHIBA is even higher than in ECHAM4/IMPL for most of the time. The reason is that the soil moisture in SECHIBA cannot be further reduced, which leads to a lower evapotranspiration and therefore less cooling. Sensitivity experiments with the same model setup, but higher initial soil moisture, have shown that because of its soil hydrology and stress formulations, SECHIBA is generally more effective in keeping the water stored in the soil under dry and medium-wet conditions than ECHAM. Therefore, SECHIBA tends to simulate higher surface temperatures under these conditions. 


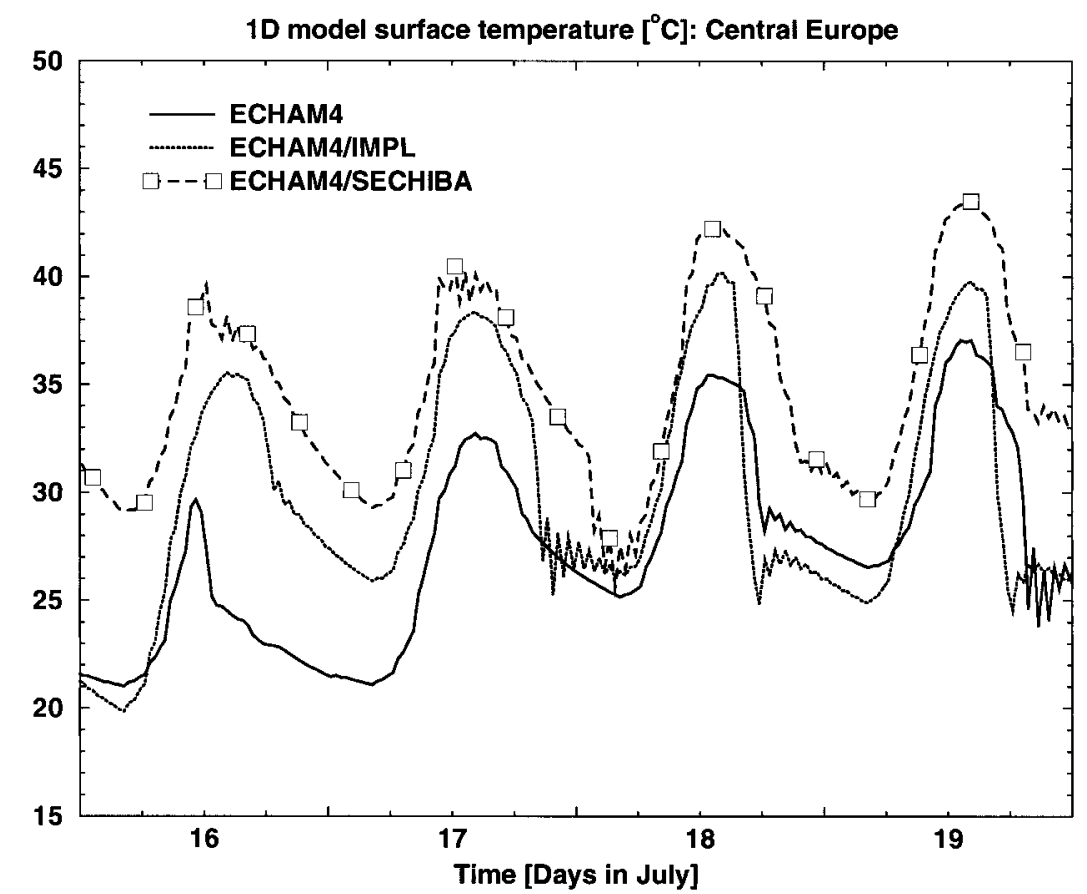

FIG. 11. Diurnal cycles of surface temperature as simulated by ECHAM4, ECHAM4/ IMPL, and ECHAM4/SECHIBA 1D from 16 to $19 \mathrm{Jul}$ at a site in central Europe.

On day 19 there is an example of a negative peak of the residual energy term in ECHAM4 of less than $-1300 \mathrm{~W} \mathrm{~m}^{-2}$ supporting a very unrealistic latent heat flux of more than $1700 \mathrm{~W} \mathrm{~m}^{-2}$ (Fig. 12). This again forces an abrupt decrease of the surface temperature, followed by some oscillations during the subsequent time steps (see Fig. 11). Similar oscillations are also visible in the two other models at different times. They are mainly due to the leapfrog time-stepping scheme used in the other physics modules of the atmospheric model, for example, the vertical diffusion scheme. It is characterized by two different alternating chains of val-

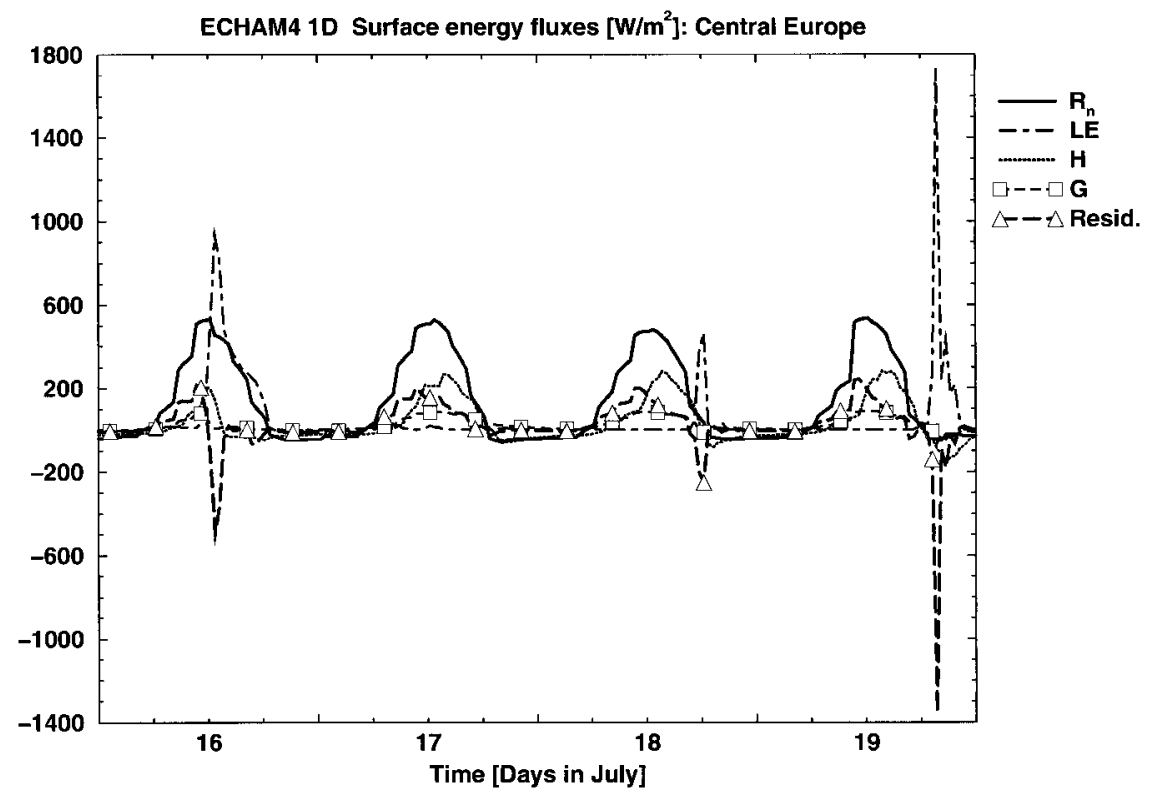

FIG. 12. Diurnal cycles of total net radiation $\left(R_{n}\right)$, latent heat flux $(L E)$, sensible heat flux $(H)$, ground heat flux $(G)$, and the surface energy residual term as simulated by ECHAM4 1D from 16 to $19 \mathrm{Jul}$ at a site in central Europe. 
ues that use a $2 \Delta t$ time step to calculate the next value, while passing the other chain. In certain situations these chains can diverge, especially after strong temperature gradients. This causes the oscillations. In order to reach a convergence of the two chains, a time filter is used to connect them.

Former versions of the ECMWF numerical weather forecast model that used a semi-implicit coupling scheme had similar problems. The ECHAM climate model was originally derived from such an ECMWF model version. Its Cycle-48 version, which includes a skin temperature scheme that is implicitly coupled to the vertical diffusion module (Viterbo and Beljaars 1995), has to deal with that as well (P. Viterbo 1996, personal communication).

An oscillation problem also emerges in the soil moisture simulation in ECHAM4, in particular in the second half of the 30-day period (cf. Fig. 10). In ECHAM4/ SECHIBA this is avoided by the use of a $1 \Delta t$ forward time-stepping scheme in the hydrologic part of the model.

As demonstrated here, the land surface schemes of all three models suffer from the effect just described, causing oscillations of the simulated surface temperature. This happens in both the semi-implicitly and the implicitly coupled models to a comparably similar extent. However, it shall be recalled that the implicit models have the great advantage of the conservation of energy.

\section{Comparison with the Cabauw observations}

It has already been discussed in the previous section that running a land surface scheme in offline mode, using an atmospheric forcing, involves a number of disadvantages. The lack of any interactions between surface and atmosphere in such a model setup significantly limits the transferability of the results to the case when the surface scheme is coupled to an atmospheric model. For instance, the role of the coupling mechanism and the evaporation-precipitation feedback found in the 1D simulation with ECHAM4 (cf. Fig. 4) could never be detected in offline experiments. They would therefore miss a model feature that is important for the understanding of the GCM behavior.

On the other hand, for an evaluation of the model results observations are needed. The 1D intercomparison of ECHAM4 and ECHAM4/IMPL has shown that a part of the energy lost to the residual term in ECHAM4 contributes to an altered diurnal cycle of the sensible heat flux in ECHAM4/IMPL (compare Figs. 2 and 5). However, without a comparison to observations it cannot be decided which one may be more realistic. In order to enable us to do this, offline experiments are a useful tool. In this section results from offline simulations with the three models, employing the Cabauw dataset (Beljaars and Bosveld 1997), are presented. As mentioned before, in offline mode atmospheric feedbacks are avoided, but still the energy balance and the surface energy fluxes are calculated using the equations given in section 3. The basic idea of this comparison is to use equivalent parameter values for soil and vegetation of each land surface scheme and to force them with the same atmospheric values. This methodology is adopted from the PILPS project, which also used the Cabauw dataset for model intercomparison purposes (cf. Chen et al. 1997; Schulz et al. 1998).

Parameters such as roughness length, leaf area index and albedo, and the initial values of the moisture reservoirs and temperatures were prescribed for all schemes in a consistent way. Each model was run for a few years applying the 1-yr forcing dataset repeatedly until equilibrium was reached.

\section{a. The Cabauw observational data}

The Cabauw site is located in the center of Netherlands (at $51^{\circ} 58^{\prime} \mathrm{N}, 4^{\circ} 56^{\prime} \mathrm{E}$ ). The flat terrain is almost completely covered with grass so that the vegetation ratio is nearly equal to 1 . This means that the soil water is almost exclusively extracted by transpiration and not by bare soil evaporation. Details about the measuring program at Cabauw are given by Driedonks et al. (1978). The set of observational data includes the meteorological quantities that are used as atmospheric forcing to drive the land surface schemes, that is, solar and thermal downward radiation, precipitation, horizontal wind components, air temperature, and specific humidity. Radiation, temperature, and humidity undergo a marked annual cycle with a maximum in summer; the wind speed has a maximum in winter. For precipitation no annual cycle is obvious. For validation, measurements of sensible and latent heat fluxes, total net radiation, surface ground heat fluxes, and soil temperature are available. The observations cover a 1-yr period (1987) at a time resolution of $30 \mathrm{~min}$. It must be noted that the three schemes were all forced with the observational data at a $30-$ min time step $\Delta t$.

Studies of the data accuracy (Beljaars and Bosveld 1997) have shown that some observed quantities of the surface energy balance were seriously biased. Therefore, while the forcing variables of the PILPS Cabauw experiment have been extracted from an earlier version of the Cabauw data (Beljaars and Viterbo 1994), the energy fluxes for model validation were derived from a version of the Cabauw data that includes bias corrections (Beljaars and Bosveld 1997). The same procedure as described above was also applied to the Cabauw data by Chen et al. (1997) for the model intercomparison in PILPS. According to Chen et al. (1997) the remaining minor inconsistencies between the forcing and the validation data can be tolerated.

\section{b. Results}

Figure 13 shows the diurnal variations of the surface temperature for a 5-day period (15-19 September as an 


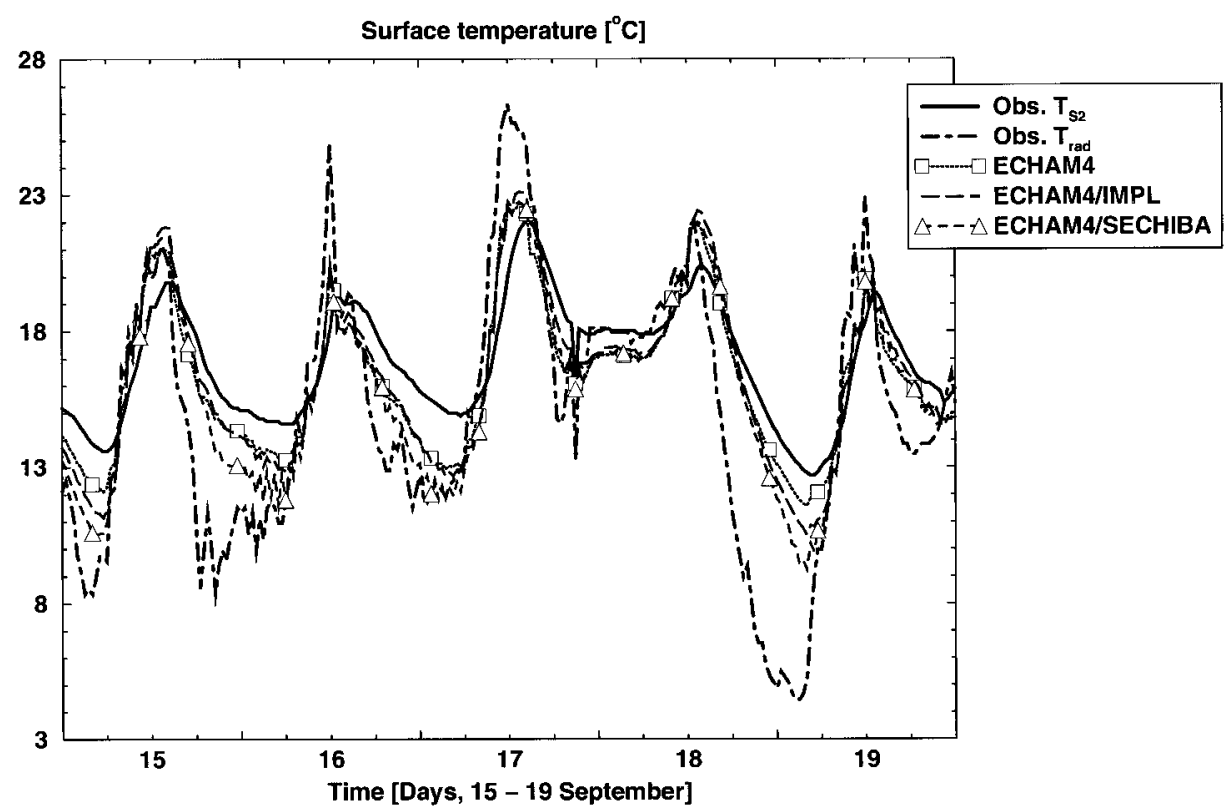

FIG. 13. Diurnal cycles of soil temperature $T_{S 2}$ measured at a depth of $2 \mathrm{~cm}$ and observed surface radiative temperature $T_{\text {rad }}$ from 15 to $19 \mathrm{Sep}$ as compared with the surface temperatures as simulated by ECHAM4, ECHAM4/IMPL, and ECHAM4/SECHIBA. The observations were made at Cabauw.

example) as observed at Cabauw and as simulated by ECHAM4, ECHAM4/IMPL, and ECHAM4/SECHIBA. Here, $T_{\text {rad }}$ is the observed surface radiative temperature, which is calculated from the observed upward longwave radiation, and $T_{S 2}$ is the soil temperature measured at a depth of $2 \mathrm{~cm}$. The diurnal cycles of the three model surface temperatures are very similar, but there is a tendency for the characteristic shape of the diurnal temperature curve of ECHAM4 to be closer to $T_{S 2}$ than that of the other model versions.

Because the ECHAM model soil temperatures are representative for the middle of each soil layer, the temperature $T_{1}$ of the top layer, which is $6.5 \mathrm{~cm}$ deep, is expected to be comparable with the measured value at a depth of $3.25 \mathrm{~cm}$ (cf. Fig. 1). The amplitude of the diurnal soil temperature variation decreases with depth because of the heat conduction (diffusion) process in the soil. In addition, there is a phase delay of the temperature signal with depth. Therefore, the amplitude of the diurnal variations of the ECHAM model temperature is expected to be smaller than that of $T_{S 2}$ but, as seen in Fig. 13, it is larger instead. The temperature curve is within the range of $T_{S 2}$ and $T_{\text {rad }}$ almost all the time. This behavior is mainly due to the discrepancy in the model that on the one hand the first model layer temperature $T_{1}$ represents the soil temperature at a depth of $3.25 \mathrm{~cm}$ according to the soil heat conduction scheme; on the other hand, it is also used as surface temperature for computing the atmospheric energy fluxes. In reality, the relevant surface temperature for these energy fluxes is the one that is "seen" by the atmosphere at the soilvegetation-atmosphere interface.
As indicated by Fig. 13, the surface temperature simulated by ECHAM4/IMPL has a larger diurnal amplitude than that of ECHAM4 and thus gets closer to the observed radiative temperature. This behavior is even more emphasized by ECHAM4/SECHIBA. The difference between ECHAM4 and ECHAM4/IMPL can be explained by the energy residual term that occurs as part of the semi-implicit coupling procedure in ECHAM4. Its diurnal evolution is shown in Fig. 14. In the morning hours until about noon it is positive, which indicates a loss of energy due to the numerics, and in the evening and night this situation is reversed, that is, energy is created. The energy residual term is generally of the order of magnitude of the ground heat flux although a number of larger peaks are found. Because the energy in ECHAM4/IMPL is conserved, this allows, for example, a more effective cooling during the night due to the absence of an artificial heating through energy created by the numerics. The even more pronounced diurnal cycle of the surface temperature in ECHAM4/ SECHIBA (Fig. 13) can be explained by the different physical parameterization, since the surface energy balance equation in SECHIBA is solved using the soil properties obtained by an extrapolation of heat capacity and ground heat flux toward the surface (cf. section $2 b$ ). The impact of this procedure can directly be seen in the simulated ground heat flux, which has a larger diurnal amplitude and is earlier in phase in ECHAM4/SECHIBA than in ECHAM4/IMPL (see Fig. 15). This is in agreement with the observations.

The semi-implicit coupling in ECHAM4 has also an unfavorable impact on the simulated turbulent heat flux- 


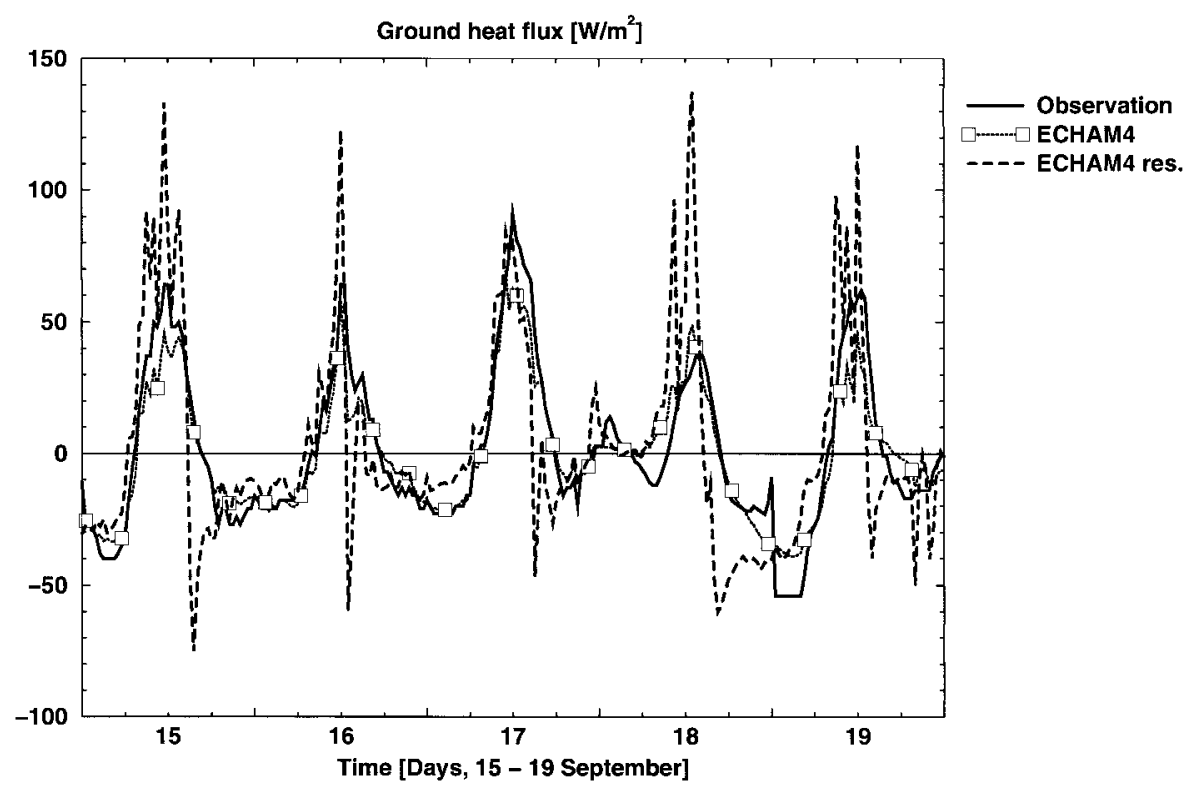

FIG. 14. Diurnal cycles of surface ground heat flux as observed at Cabauw from 15 to 19 Sep as compared with the model results of ECHAM4. ECHAM4 res. denotes the surface energy residual in ECHAM4.

es (cf. Fig. 16 for the sensible heat flux). They tend to be underestimated, and their rise in the morning and decrease in the afternoon are delayed by up to several hours, which leads to a phase shift of their diurnal cycles. Both effects are mainly caused by the energy residual term. Because of the improved coupling, includ- ing the more consistent formulation of the turbulent heat fluxes, the simulated fluxes of the implicit models are in better agreement with the Cabauw observations, especially regarding the phases of their diurnal cycles.

Another way of looking at the results is scatterplots of simulated versus observed values of the same quan-

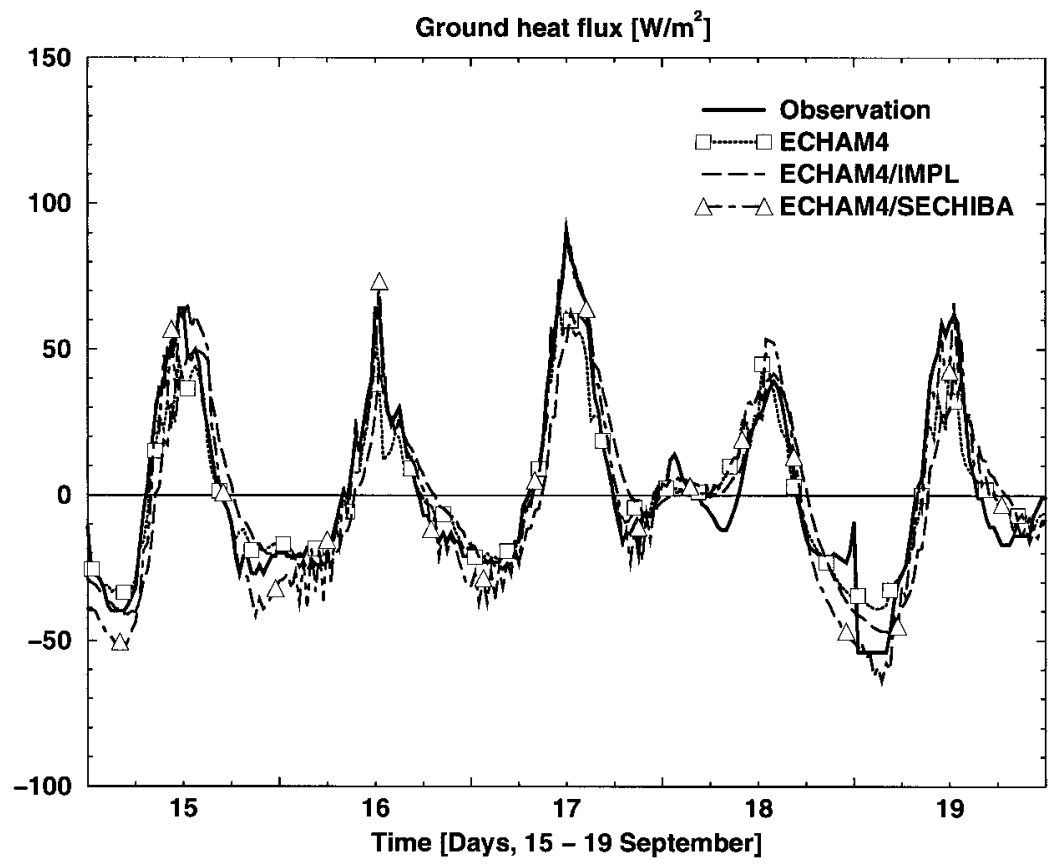

FIG. 15. Diurnal cycles of surface ground heat flux as observed at Cabauw from 15 to 19 Sep as compared with the model results of ECHAM4, ECHAM4/IMPL, and ECHAM4/ SECHIBA. 


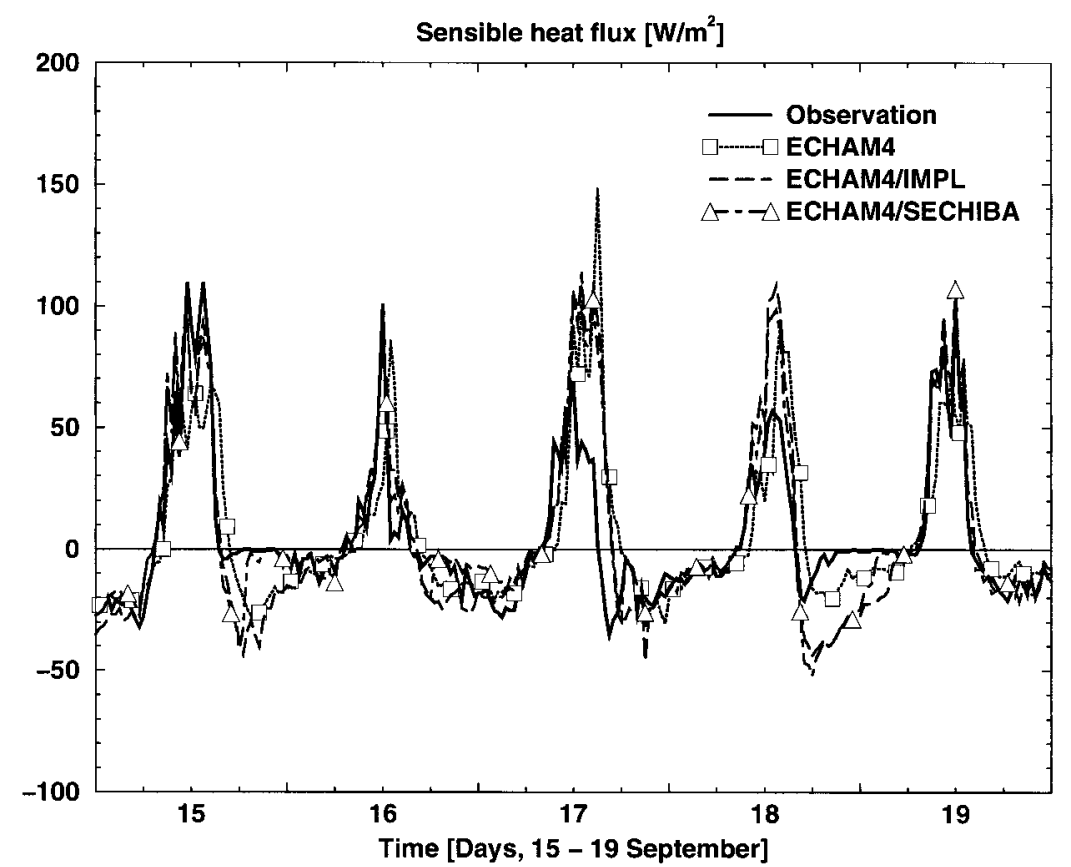

FIG. 16. As Fig. 15 but for sensible heat flux.
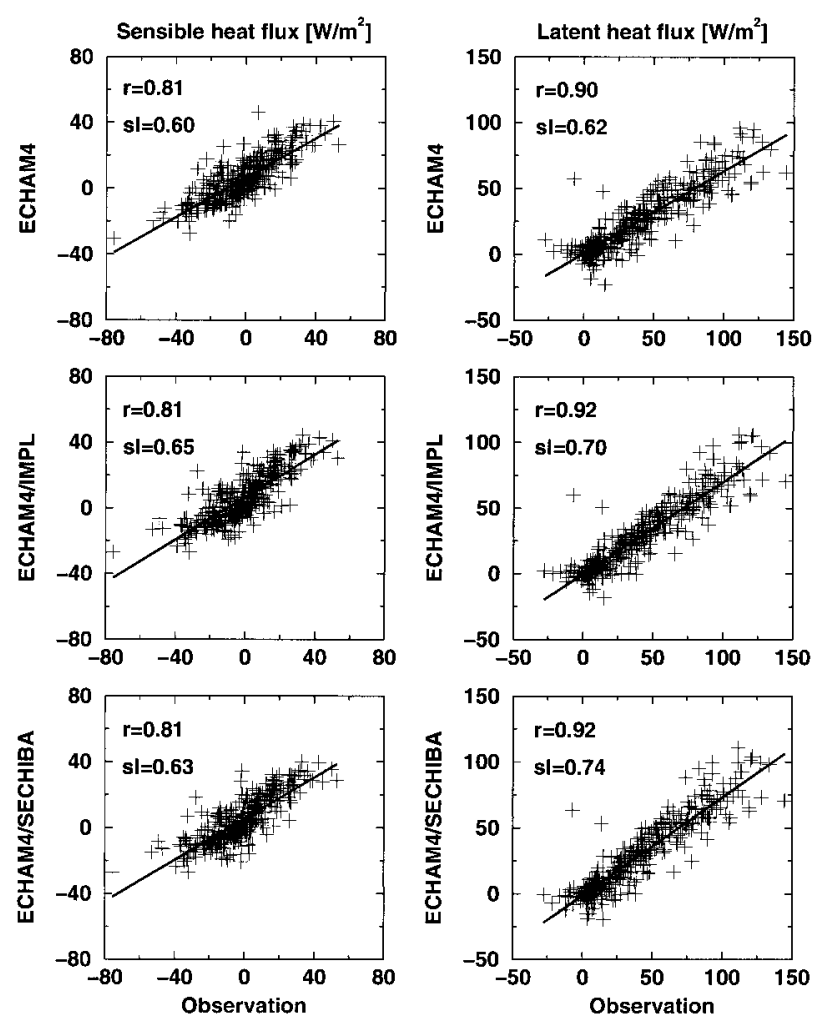

FIG. 17. Scatterplots of simulated vs observed daily mean sensible and latent heat fluxes for ECHAM4, ECHAM4/IMPL, and ECHAM4/ SECHIBA. The plots cover the entire 1-yr simulation period. The observations were made at Cabauw. The correlation coefficients $r$ and the slopes sl of the regression curves are indicated. All panels show a good correlation between model and observation. tity. Figure 17 shows such scatterplots for the daily mean sensible and latent heat fluxes for the entire 1-yr simulation period of the three models. All three models tend to underestimate latent heat flux, in particular the high values, and overestimate sensible heat flux, especially in the range of low and negative values. This is in agreement with the findings by Schulz et al. (1998). Therefore, the slopes of the linear regression curves in Fig. 17 are all significantly smaller than 1 , being lowest for ECHAM4. Here, the effect of energy loss due to the semi-implicit coupling can be seen. The annual mean value of the energy residual in the ECHAM4 simulation amounts to $2.6 \mathrm{~W} \mathrm{~m}^{-2}$. Daily mean values peak between -40 and almost $30 \mathrm{~W} \mathrm{~m}^{-2}$. In ECHAM4/IMPL the regression curves for both sensible and latent heat flux are steeper, which is mainly attributed to a better representation of the diurnal cycles and the energy conservation in the implicit model. Because of its different parameterization of evaporation, ECHAM4/SECHIBA allows for a higher latent heat flux in summer; the sensible heat flux is reduced accordingly. Both fluxes are closer to the observations than in ECHAM4/IMPL. The scatterplots indicate that, here, improving the numerical coupling is at least as or more important for improving the simulation than changing the physical parameterization.

In all, this shows that solving the surface energy balance equation with an implicit method that prevents energy loss or creation by the numerics, in combination with a model structure as in SECHIBA (here in particular the extrapolation of heat capacity and ground heat flux toward the surface), yields more realistic surface heat fluxes. 


\section{Conclusions}

The 1D versions of the standard ECHAM4 GCM and the two modified models ECHAM4/IMPL and ECHAM4/SECHIBA were compared. In ECHAM4, land surface and atmosphere are coupled semi-implicitly in a way that the energy balance at the land surfaceatmosphere interface is not closed. The two modified models incorporate an implicit coupling technique that has as one major advantage the conservation of energy at the land surface. The implicit and semi-implicit coupling techniques are standard methodologies used in current GCMs.

ECHAM4 and ECHAM4/IMPL are identical with respect to all physical parameterizations they apply; the only difference is the coupling. In ECHAM4/SECHIBA the ECHAM land surface scheme was replaced by the full SECHIBA land surface scheme. Therefore, this set of three different models allows a systematic analysis of the impact of both the numerical coupling and the physical parameterization on the simulated climate.

A 1D simulation for July at a central European site under prevailing dry conditions was performed with each of the three models, using the same initialization and an equivalent set of surface parameters. Particular emphasis has been laid on analyzing the behavior of the energy residual term in ECHAM4, which is part of the semi-implicit coupling and represents an error in the surface-atmosphere energy balance. It was found not to be negligibly small, but rather to be of an order of magnitude similar to the physical fluxes of, for example, latent or sensible heat. This term serves as an artificial (numerical) sink or source of energy at the surface that may significantly alter the surface energy balance. Because of this, unrealistic high latent heat flux values of more than $1700 \mathrm{~W} \mathrm{~m}^{-2}$ were found in the simulation. Only a very little portion of this energy may be provided by other physical terms of the energy balance. For instance, in this particular case, more than $1300 \mathrm{~W} \mathrm{~m}^{-2}$ were compensated by the energy residual. This had also a disadvantageous effect for the convective precipitation that was triggered by the evaporation, associated with the latent heat flux. This model behavior represents a clear deficiency in ECHAM4 and shows that great care needs to be taken in the choice of numerical methods applied. In the case of a GCM, this means that, besides other things, the numerical coupling between surface and atmosphere needs to be designed properly to ensure that the surface energy balance can be realistically calculated. Because the energy and water cycles are coupled via the latent heat flux or evapotranspiration, respectively, a proper numerical treatment has important implications also for the surface moisture balance.

These results were compared with ECHAM4/IMPL, which utilizes an implicit coupling technique with a closed land surface-atmosphere energy balance. Here, the energy, which was lost to (or created by) the numerical scheme in ECHAM4, is conserved for the phys- ical processes. Similar to ECHAM4, a positive feedback between convective precipitation and canopy evaporation occurs in the July simulation, but here the energy available for the latent heat flux is more realistically controlled. An extreme overestimation of latent heat flux (and associated precipitation) peaks and an energy loss due the numerics, such as in ECHAM4, are avoided in ECHAM4/IMPL. This was found to result in a more pronounced diurnal cycle of surface temperature and generally higher temperature maxima during a warming phase.

Furthermore, a conceptual inconsistency in the ECHAM parameterization was identified that allows the removal of additional water from the soil when required, which occurs whenever the calculated canopy evaporation exceeds the available water in the interception reservoir. This was found to lead to a reduction of the soil water content below the wilting level by transpiration, which should not be possible by definition of the wilting point. In SECHIBA such an effect is avoided by a predictor-corrector method, which reduces the canopy evaporation accordingly to the available water on the canopy. In addition, because of its soil hydrology and stress formulations, SECHIBA was found to be generally more effective than ECHAM in keeping the water stored in the soil under dry conditions. Therefore, SECHIBA tends to simulate higher surface temperatures under these conditions. Besides that, SECHIBA additionally allows some bare soil evaporation even under prevailing dry conditions, as a result of a different formulation of the soil moisture stress. This may occur as a response to heavy rain showers when the upper soil moisture layer, which is part of the Choisnel soil hydrologic scheme, is filled. This was found to be a reasonable approach.

Numerical instabilities in the calculation of the surface temperature, which occur in ECHAM4 because of the leapfrog time-stepping scheme in certain situations with associated rapid surface temperature changes, were found not to be avoided by the implicit coupling. They occur to a similar extent, but one has to keep in mind that the implicit models offer the important advantage of conserving energy at the land surface-atmosphere interface.

The three models were also tested against observations. This was done in offline mode, using observational data from Cabauw (Netherlands) as atmospheric forcing for the land surface schemes. A 1-yr simulation was performed with the three models, using the same initialization and equivalent surface parameter values. The offline results confirm the findings of the 1D experiments in that the coupling technique has an important impact on the simulated surface energy cycle, favoring the more realistic implicit coupling method when comparing with the observations. In particular, because of the energy residual term, which occurs as part of the semi-implicit coupling procedure in ECHAM4, the turbulent heat fluxes tend to be underestimated; their rise 
in the morning and decrease in the afternoon are delayed. Because of the improved (energy conserving) coupling, the turbulent heat fluxes of the implicit models are in better agreement with the Cabauw observations, especially regarding the phases of their diurnal cycles.

In SECHIBA the surface energy balance equation is solved using the soil properties obtained by an extrapolation of heat capacity and ground heat flux toward the surface. It was found that because of this in ECHAM4/ SECHIBA the diurnal cycle of the ground heat flux has a larger amplitude and is earlier in phase than in ECHAM4/IMPL, which is in agreement with the observations. Furthermore, the simulated surface temperature of ECHAM4/SECHIBA gets closer to the observed radiative temperature for the latter model. Therefore, it is concluded that solving the surface energy balance equation with an implicit technique, which prevents energy loss or creation by the numerics (in contrast to a semi-implicit method), in combination with the particular model structure in SECHIBA yields more realistic surface heat fluxes.

It is further concluded that for a comprehensive assessment of a land surface scheme, which may allow one to estimate its influence on the simulated climate when implemented in a GCM, it is indispensible to analyze it in a coupled mode as in a one-dimensional model. Only then it is possible to capture such important model features as surface-atmosphere interactions, including feedbacks. An offline experiment is a useful tool for investigating the land surface scheme behavior under well-defined restricted conditions. However, one has to keep in mind its limitations, which prevent the immediate use of its findings for the interpretation of GCM results.

For future offline intercomparison studies such as in PILPS, new methods for forcing land surface schemes need to be devised that take into account the coupling to the atmosphere. If this is not done we will find, as in this study, that the offline evaluation of these schemes will not be relevant for the coupled environment. Schemes that might look favorable when forced by observations can turn out to be unacceptable for GCMs, because their coupling methods may induce systematic biases and incorrect feedbacks in the atmosphere.

As consequent extension of the intercomparison of the 1D versions of the three GCMs, ECHAM4, ECHAM4/IMPL, and ECHAM4/SECHIBA, in this study, an analysis of the models' behavior in $3 \mathrm{D}$ global experiments will be presented in a forthcoming paper.

Acknowledgments. We acknowledge the Royal Netherlands Meteorological Institute (KNMI) for providing the Cabauw observational data. We are very grateful to Adrian Tompkins, Roman Hatzky, Pedro Viterbo, and Anton Beljaars for many helpful discussions. We would like to extend our gratitude to Uwe Schulzweida, Uli Schlese, and Monika Esch for continuous support in all technical aspects, and Norbert Noreiks for assistance in creating some of the figures.

\section{APPENDIX}

\section{Numerical Scheme for the Vertical Diffusion}

The turbulent exchange of heat and humidity at the surface and their turbulent vertical transport in the lower atmosphere are described as a diffusive process. The equation for the vertical diffusion that is solved in the ECHAM4 model is

$$
\begin{aligned}
\frac{\partial X(z, t)}{\partial t} & =\frac{1}{\rho(z, t)} \frac{\partial}{\partial z}\left[\rho(z, t) K(z, t) \frac{\partial X(z, t)}{\partial z}\right] \\
& =\frac{1}{\rho(z, t)} \frac{\partial J_{X}(z, t)}{\partial z},
\end{aligned}
$$

where $X$ may be identified with the dry static energy $s$ or the specific humidity $q$. Here, $K$ is the exchange coefficient and $J_{X}$ (positive downward) is the vertical turbulent flux of $X ; \rho$ is the air density, $t$ is time, and $z$ is the vertical coordinate.

In order to solve (A1) over the entire atmospheric column, numerical boundary conditions are needed. At the top of the planetary boundary layer a zero flux condition is chosen. At the surface, bulk formulas for the surface sensible and latent heat fluxes as given by (3), which take into account the surface properties, are introduced for $J_{X}$. Thus, the entire vertical turbulent transport including the surface fluxes can be computed with (A1). This shows how closely linked the land surface and the vertical diffusion schemes are.

In ECHAM4 (A1) is solved numerically using a discretization in the vertical and in time. Figure A1 gives a schematic representation of the vertical discretization. It shall be assumed that the computation is performed from the $T$ th atmospheric level (top) to the $N$ th level (bottom). Variables are calculated at full levels (solid lines) and fluxes at intermediate levels (dashed lines). Here, $X_{S}$ denotes the surface value of $X$. Equation (A1) is solved implicitly using a leapfrog time-stepping scheme. The computation starts at the previous time step, denoted as $t-1$, when all variables are known. The equation is solved with explicit coefficients, which means that the values of $\rho$ and $K$ at time $t-1$ are used. The system is integrated over a $2 \Delta t$ time step ( $1 \Delta t$ at the first time step), yielding the new values at time $t+$ 1. The discretization of (A1) results in the following finite-difference scheme for level $k(k=T, T+1, \ldots$, $N)$ :

$$
\begin{aligned}
\frac{X_{k}^{t+1}-X_{k}^{t-1}}{2 \Delta t}=\frac{g}{\Delta p_{k}}\left(\rho_{k+1 / 2}^{t-1} K_{k+1 / 2}^{t-1} \frac{X_{k+1}^{*}-X_{k}^{*}}{\Delta z_{k+1 / 2}}\right. & \\
& \left.\quad-\rho_{k-1 / 2}^{t-1} K_{k-1 / 2}^{t-1} \frac{X_{k}^{*}-X_{k-1}^{*}}{\Delta z_{k-1 / 2}}\right)
\end{aligned}
$$




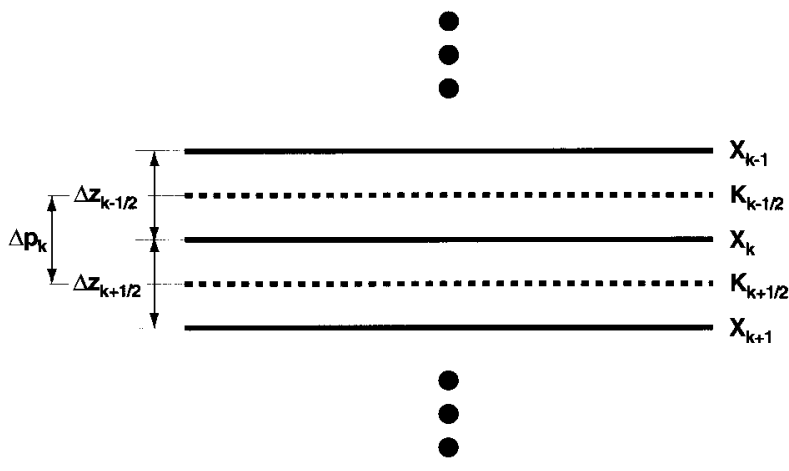

FIG. A1. Schematic representation of the full and intermediate levels used for the discretization of the vertical diffusion equation.

$g$ is the acceleration of gravity of the earth, and $\Delta p_{k}$ is the pressure difference between two adjacent intermediate levels; both are induced here through the hydrostatic equation. Here, $X_{k}^{*}$ is given by

$$
X_{k}^{*}=\alpha X_{k}^{t+1}+(1-\alpha) X_{k}^{t-1} \quad \text { with } \alpha=1.5 \text {. }
$$

Equation (A2) can be written as

$$
\begin{aligned}
& -\frac{A_{k+1 / 2}^{t-1}}{\Delta p_{k}} \frac{X_{k+1}^{*}}{\alpha}+\left(1+\frac{A_{k+1 / 2}^{t-1}}{\Delta p_{k}}+\frac{A_{k-1 / 2}^{t-1}}{\Delta p_{k}}\right) \frac{X_{k}^{*}}{\alpha}-\frac{A_{k-1 / 2}^{t-1}}{\Delta p_{k}} \frac{X_{k-1}^{*}}{\alpha} \\
& =\frac{X_{k}^{t-1}}{\alpha},
\end{aligned}
$$

when using the definition

$$
A_{k+1 / 2}^{t-1}=2 \Delta \operatorname{t\alpha g} \frac{\rho_{k+1 / 2}^{t-1} K_{k+1 / 2}^{t-1}}{\Delta z_{k+1 / 2}} .
$$

Rewriting of (A4) leads to

$$
-\hat{A}_{k+1 / 2}^{t-1} \frac{X_{k+1}^{*}}{\alpha}+\hat{B}_{k+1 / 2}^{t-1} \frac{X_{k}^{*}}{\alpha}-\hat{C}_{k+1 / 2}^{t-1} \frac{X_{k-1}^{*}}{\alpha}=\frac{X_{k}^{t-1}}{\alpha}
$$

with the coefficients

$$
\begin{aligned}
& \hat{A}_{k+1 / 2}^{t-1}=\frac{A_{k+1 / 2}^{t-1}}{\Delta p_{k}}, \quad \hat{B}_{k+1 / 2}^{t-1}=\left(1+\frac{A_{k+1 / 2}^{t-1}}{\Delta p_{k}}+\frac{A_{k-1 / 2}^{t-1}}{\Delta p_{k}}\right), \\
& \hat{C}_{k+1 / 2}^{t-1}=\frac{A_{k-1 / 2}^{t-1}}{\Delta p_{k}} .
\end{aligned}
$$

This system of equations is solved using an algorithm proposed by Richtmyer and Morton (1967). It allows one to reduce the system (A6) to a set of equations, of the form

$$
\frac{X_{k}^{*}}{\alpha}=E_{k+1 / 2}^{t-1} \frac{X_{k+1}^{*}}{\alpha}+F_{k+1 / 2}^{t-1}
$$

that can be explicitly solved (see below). The coefficients $E_{k+1 / 2}^{t-1}$ and $F_{k+1 / 2}^{t-1}$ are given by

$$
\begin{aligned}
E_{k+1 / 2}^{t-1} & =\frac{\hat{A}_{k+1 / 2}^{t-1}}{\hat{B}_{k+1 / 2}^{t-1}-\hat{C}_{k+1 / 2}^{t-1} E_{k-1 / 2}^{t-1}} \text { and } \\
F_{k+1 / 2}^{t-1} & =\frac{X_{k}^{t-1} / \alpha+\hat{C}_{k+1 / 2}^{t-1} F_{k-1 / 2}^{t-1}}{\hat{B}_{k+1 / 2}^{t-1}-\hat{C}_{k+1 / 2}^{t-1} E_{k-1 / 2}^{t-1}} .
\end{aligned}
$$

As the coefficients of (A2) are explicit, this is also true for (A4) and (A6). Therefore, $E_{k+1 / 2}^{t-1}$ and $F_{k+1 / 2}^{t-1}$ are calculated at time $t-1$. From these equations together with the zero flux condition at the top (i.e., $\hat{C}_{T+1 / 2}=0$ ), one can calculate the $E_{k+1 / 2}$ and $F_{k+1 / 2}$ iteratively from top to bottom of the vertical column, that is in order of increasing $k(k=T, T+1, \ldots, N)$. For $k=N$ in (A7), there is a value needed for $X_{N+1}^{*}$. This is the surface value of $X$. Depending on the type of coupling between land surface scheme and atmosphere, the value at time $t-1$ or $t+1$ will be used (see section 3 ). Using this boundary condition at the bottom, the $X_{k}^{*}$ can be calculated iteratively from (A7) in order of decreasing $k$ $(k=N, N-1, \ldots, T)$. This back substitution yields the vertical profile of $X$ at the new time step.

\section{REFERENCES}

Beljaars, A. C. M., and P. Viterbo, 1994: The sensitivity of winter evaporation to the formulation of aerodynamic resistance in the ECMWF model. Bound.-Layer Meteor., 71, 135-149.

- , and F. C. Bosveld, 1997: Cabauw data for the validation of land surface parameterization schemes. J. Climate, 10, 11721193.

Blondin, C., 1991: Parameterization of land surface processes in numerical weather prediction. Land Surface Evaporation: Measurements and Parameterization, T. J. Schmugge and J. C. Andre, Eds., Springer-Verlag, 31-54.

Charney, J. G., W. J. Quirk, S.-H. Chow, and J. Kornfield, 1977: A comparative study of the effects of albedo change on drought in semi-arid regions. J. Atmos. Sci., 34, 1366-1385.

Chen, T. H., and Coauthors, 1997: Cabauw experimental results from the Project for Intercomparison of Land-surface Parameterization Schemes. J. Climate, 10, 1194-1215.

Choisnel, E., 1977: Le bilan d'énergie et le bilan hydrique du sol. La Météorologie, VI, 11, 103-133.

Christensen, J. H., and E. van Meijgaard, 1992: On the construction of a regional atmospheric climate model. Royal Netherlands Meteorological Institute Tech. Rep. 147, 22 pp.

- O. B. Christensen, P. Lopez, E. van Meijgaard, and M. Botzet, 1996: The HIRHAM4 regional atmospheric climate model. Danish Meteorological Institute Sci. Rep. 96-4, 51 pp.

Déqué, M., P. Marquet, and R. G. Jones, 1998: Simulation of climate change over Europe using a global variable resolution general circulation model. Climate Dyn., 14, 173-189.

Dickinson, R. E., A. Henderson-Sellers, P. J. Kennedy, and M. F. Wilson, 1986: Biosphere-Atmosphere Transfer Scheme (BATS) for the NCAR Community Climate Model. National Center for Atmospheric Research Tech. Note NCAR/TN-275 + STR, 69 pp.

Driedonks, A. G. M., H. van Dop, and W. Kohsiek, 1978: Meteorological observations on the $213 \mathrm{~m}$ mast at Cabauw in the Netherlands. Proc. Fourth Symp. on Meteorological Observations and Instrumentation, Denver, CO, Amer. Meteor. Soc., 4146.

Ducoudré, N. I., K. Laval, and A. Perrier, 1993: SECHIBA, a new set of parameterizations of the hydrologic exchanges at the landatmosphere interface within the LMD atmospheric general circulation model. J. Climate, 6, 248-273. 
Dümenil, L., and E. Todini, 1992: A rainfall-runoff scheme for use in the Hamburg climate model. Advances in Theoretical Hydrology: A Tribute to James Dooge, J. P. O'Kane, Ed., Elsevier Science Publishers, 129-157.

Henderson-Sellers, A., K. McGuffie, and A. J. Pitman, 1996: The Project for Intercomparison of Land-Surface Parameterization Schemes (PILPS): 1992 to 1995. Climate Dyn., 12, 849-859.

Jones, R. G., J. M. Murphy, and M. Noguer, 1995: Simulation of climate change over Europe using a nested regional-climate model. I: Assessment of control climate including sensitivity to location of lateral boundaries. Quart. J. Roy. Meteor. Soc., 121, 1413-1449.

Louis, J.-F., 1979: A parametric model of vertical eddy fluxes in the atmosphere. Bound.-Layer Meteor., 17, 187-202.

Manabe, S., 1969: Climate and the ocean circulation. I. The atmospheric circulation and the hydrology of the earth's surface. Mon Wea. Rev., 97, 739-774.

Milly, P. C. D., and K. A. Dunne, 1994: Sensitivity of the global water cycle to the water-holding capacity of land. J. Climate, 7, 506-526.

Peylin, P., J. Polcher, G. Bonan, D. L. Williamson, and K. Laval, 1997: Comparison of two complex land surface schemes coupled to the National Center for Atmospheric Research general circulation model. J. Geophys. Res., 102, 19 413-19 431.

Polcher, J., and Coauthors, 1998: A proposal for a general interface between land surface schemes and general circulation models. Global Planet. Change, 19, 261-276.

Richtmyer, R. D., and K. W. Morton, 1967: Difference Methods for Initial-Value Problems. 2d ed. Interscience Publishers, 405 pp.

Roeckner, E., and Coauthors, 1992: Simulation of the present-day climate with the ECHAM model: Impact of model physics and resolution. Max-Planck-Institut für Meteorologie Rep. 93, 171 pp.
- and Coauthors, 1996: The atmospheric general circulation model ECHAM4: Model description and simulation of present-day climate. Max Planck-Institut für Meteorologie Rep. 218, 90 pp.

Sato, N., P. J. Sellers, D. A. Randall, E. K. Schneider, J. Shukla, J. L. Kinter III, Y.-T. Hou, and E. Albertazzi, 1989: Effects of implementing the Simple Biosphere model in a general circulation model. J. Atmos. Sci., 46, 2757-2782.

Schulz, J.-P., L. Dümenil, J. Polcher, C. A. Schlosser, and Y. Xue, 1998: Land surface energy and moisture fluxes: Comparing three models. J. Appl. Meteor., 37, 288-307.

Sellers, P. J., Y. Mintz, Y. C. Sud, and A. Dalcher, 1986: A Simple Biosphere Model ( $\mathrm{SiB}$ ) for use within general circulation models. J. Atmos. Sci., 43, 505-531.

Shukla, J., and Y. Mintz, 1982: Influence of land-surface evapotranspiration on the earth's climate. Science, 215, 1498-1501.

Stamm, J. F., E. F. Wood, and D. P. Lettenmaier, 1994: Sensitivity of a GCM simulation of global climate to the representation of land surface hydrology. J. Climate, 7, 1218-1239.

Stull, R. B., 1997: An Introduction to Boundary Layer Meteorology. Kluwer Academic, 670 pp.

Sud, Y. C., J. Shukla, and Y. Mintz, 1988: Influence of land surface roughness on atmospheric circulation and precipitation: A sensitivity study with a general circulation model. J. Appl. Meteor., 27, 1036-1054.

van Lipzig, N. P. M., E. van Meijgaard, and J. Oerlemans, 1999: Evaluation of a regional atmospheric model using measurements of surface heat exchange processes from a site in Antarctica. Mon. Wea. Rev., 127, 1994-2011.

Viterbo, P., and A. C. M. Beljaars, 1995: An improved land surface parameterization scheme in the ECMWF model and its validation. J. Climate, 8, 2716-2748.

Warrilow, D. A., A. B. Sangster, and A. Slingo, 1986: Modelling of land surface processes and their influence on European climate. U.K. Met. Office Tech. Note DCTN 38, 92 pp. 